\title{
Two-grid economical algorithms for parabolic integro-differential equations with nonlinear memory
}

\author{
Wansheng Wanga ${ }^{\mathrm{a}}$, Qingguo Hong ${ }^{\mathrm{b}, *}$ \\ ${ }^{a}$ Department of Mathematics, Shanghai Normal University, Shanghai, 200234, China \\ ${ }^{b}$ Department of Mathematics, Pennsylvania State University, State College, PA \\ 16802,U.S.A.
}

\begin{abstract}
In this paper, several two-grid finite element algorithms for solving parabolic integro-differential equations (PIDEs) with nonlinear memory are presented. Analysis of these algorithms is given assuming a fully implicit time discretization. It is shown that these algorithms are as stable as the standard fully discrete finite element algorithm, and can achieve the same accuracy as the standard algorithm if the coarse grid size $H$ and the fine grid size $h$ satisfy $H=O\left(h^{\frac{r-1}{r}}\right)$. Especially for PIDEs with nonlinear memory defined by a lower order nonlinear operator, our two-grid algorithm can save significant storage and computing time. Numerical experiments are given to confirm the theoretical results.
\end{abstract}

Keywords: parabolic integro-differential equation, two-grid method, error estimate, finite element method, stability, backward Euler scheme 2008 MSC: 65M60, 65R20, 65L05

\section{Introduction}

The main purpose of this paper is to present some discretization techniques based on two finite element subspaces for solving parabolic integro-

\footnotetext{
*Corresponding author

Email addresses: w.s.wang@163.com (Wansheng Wang), huq11@psu.edu (Qingguo Hong)
} 
differential equations (PIDEs) with nonlinear memory:

$$
\begin{aligned}
& u_{t}+A u+\int_{0}^{t} K(t-s) B u(s) d s=f(x, t), \quad(x, t) \in \Omega \times(0, T], \\
& u(x, t)=0, \quad(x, t) \in \partial \Omega \times(0, T] \\
& u(x, 0)=u_{0}(x), \quad x \in \Omega
\end{aligned}
$$

where $\Omega \subset \mathbb{R}^{d}(d \geq 1)$ is a bounded and polyhedral domain with a piecewise smooth boundary $\partial \Omega, K(t)$ is a smooth or nonsmooth memory kernel, and $f$ is a known function, $u$ is the solution we need to solve which is scalar function . $A$ is a symmetric positive definite second-order elliptic operator with smooth coefficients in $x$ and $t$, and $B$ is a nonlinear operator of at most second order; that is,

$$
B u=-\nabla \cdot(\alpha(x, u) \nabla u+\beta(x, u))+\gamma(x, u) \cdot \nabla u+g(x, u) .
$$

For brevity, we will drop the dependence of variable $x$ in $\alpha(x, u), \beta(x, u)$, $\gamma(x, u)$, and $g(x, u)$ in the following exposition. We assume that the functions $\alpha(u) \in \mathbb{R}^{d \times d}$ is a tensor function, $\left.\beta(u)\right) \in \mathbb{R}^{d}, \gamma(u) \in \mathbb{R}^{d}$ are vector functions, and $g(u) \in \mathbb{R}^{1}$ is scalar function, respectively. And all the functions $\alpha(u)$, $\beta(u), \gamma(u)$ and $g(u)$ are smooth and bounded together with the Gateaux derivative. For the functions $\beta(u)$ and $g(u)$, we also assume that $\beta(0)=0$ and $g(0)=0$.

Equations of the above type, or linear versions thereof, can arise from many physical processes in which it is necessary to take into account the effects of memory due to the deficiency of the usual diffusion equations [20, 33, 39]. For approximating the solution $u$ of PIDEs, both finite difference and finite element methods have been investigated extensively in the past for both the linear and nonlinear problem (see, for example, [8, 9, 29, 31, 37, 12, 54]). Recently, several new numerical methods such as mixed finite element method, finite volume element method, and discontinuous Galerkin method for space discretization or time discretization have been proposed to solve PIDEs (see, for example, [19, 42, 36, 41, 6, 35]).

The two-grid method based on two finite element spaces, one on a coarse grid and one on a fine grid, was first developed by Xu [47, 48, 49, 50] for nonsymmetric linear and nonlinear elliptic problems. Since then, the two-grid method for elliptic problems has been investigated further, e.g., Axelsson and Layton [3], Xu and Zhou [51], Li and Huang [28], and Bi and Ginting [4, 5]. 
In these works, theoretical study and numerical experiments show that the combined use of the numerical method such as finite element method and finite difference method, and the two-grid technique is computationally more efficient than the original method. Due to this better practical performance, the two-grid method has been widely applied to the study of eigenvalue problems [52, 53, 24], steady Navier Stokes equations [27, 21, 23, 15], the timedependent Navier Stokes problem [22, 1, 2, 40, 44], the nonlinear parabolic problem [16, 32, 17, 10, 46, 14, 13, 38], and nonlinear hyperbolic equations [11]. Recently, Jin, Shu, and Xu [26] used this technique to solve decoupling systems of partial differential equations; $\mathrm{Mu}$ and Xu 34] and Cai, Mu, and Xu [7] employed it for the mixed Stokes-Darcy model. In [45], we proposed the two-grid algorithms based on the backward Euler scheme and finite element approximation for semi-linear PIDEs, and studied the long-time stability and error estimates of the two-grid algorithms.

In this paper, we present some two-grid algorithms for PIDEs with nonlinear memory and perform theoretical analysis that demonstrates our methods' ability to match the accuracy of the classic finite element method by (1) solving a nonlinear problem on a coarse space $S_{H}$ and (2) solving a symmetric positive definite linear problem on the fine space $S_{h}$. Thus, solving PIDEs with nonlinear memory is not much more difficult than solving one linear problem, as $\operatorname{dim} S_{H} \ll \operatorname{dim} S_{h}$ and the work involved in solving the nonlinear problem on the coarse grid is relatively limited.

It is worth adding that when $\alpha \equiv 0$, our algorithm significantly reduces computational memory and storage requirements. A practical difficulty of numerical methods for PIDEs is that all previous values must be stored, as they all enter subsequent equations. In order to reduce memory requirements, some economical schemes have been proposed (for example, see, [43, 25]). However, these schemes either require more regularities on the solution $u$ [43], or they cannot be applied to nonlinear problem [25].

The remainder of this article is organized as follows: In Section 2, we present some conventions and notations that will be used throughout the article. In Section 3, the stability and error estimate of the classic fully discrete finite element method are discussed. The two-grid algorithms for PIDEs with nonlinear memory are presented and the stability and error estimates of these algorithms are discussed in Section 4. In Section 5, we offer some concluding remarks.

Throughout this paper, we use the letters $C$ and $c$ (with and without subscripts) to denote a generic positive constant that stand for different values 
depending on the context in different equations. When it is not important to keep track of these constants, we conceal the letter $C$ or $c$ in the notation $\lesssim$ or $\gtrsim$, such that $x \lesssim y$ means $x \leq C y$ and $x \gtrsim y$ means $x \geq c y$.

\section{Preliminaries}

For any non-negative integer $r$ and number $p \geq 1$, let $\mathcal{W}^{r, p}(\Omega)$ be the standard Sobolev space with a norm $\|\cdot\|_{r, p}$ given by $\|v\|_{r, p}^{p}=\sum_{|\kappa| \leq r}\left\|D^{\kappa} v\right\|_{\mathcal{L}^{p}(\Omega)}^{p}$ (with the usual modification if $p=\infty$ ). This Sobolev space is also equipped with the seminorm $|v|_{r, p}^{p}=\sum_{|\kappa|=r}\left\|D^{\kappa} v\right\|_{\mathcal{L}^{p}(\Omega)}^{p}$. For $p=2$, we denote $\mathcal{H}^{r}=$ $\mathcal{W}^{r, 2}(\Omega)$ and take $\mathcal{H}_{0}^{1}$ as the subspace of $\mathcal{H}^{1}$ consisting of functions with a vanishing trace on $\partial \Omega$. For simplicity, we also use notations $\|\cdot\|_{r},\|\cdot\|$ and $\|\cdot\|_{\infty}$, and $|\cdot|_{r}$ such that $\|\cdot\|_{r}=\|\cdot\|_{r, 2},\|\cdot\|=\|\cdot\|_{0,2}$ and $\|\cdot\|_{\infty}=\|\cdot\|_{0, \infty}$, and $|\cdot|_{r}=|\cdot|_{r, 2}$.

Let $\left\{S_{h}\right\}_{0<h \leq 1}$ be a family of finite-dimensional subspaces of $\mathcal{H}_{0}^{1}$, with the following approximation properties:

$$
\inf _{\chi \in S_{h}}\left\{\|v-\chi\|+h\|v-\chi\|_{1}\right\} \lesssim h^{r}\|v\|_{r}, \quad v \in \mathcal{H}^{r} \cap \mathcal{H}_{0}^{1}, \quad r \geq 1+\frac{d}{2}
$$

We also assume that $\left\{S_{h}\right\}_{0<h \leq 1}$ satisfies the inverse hypothesis: there exists a constant $C>0$ independent of $h$ such that

$$
\|\nabla \chi\|_{\infty} \leq C h^{-d / 2}\|\nabla \chi\|, \quad \chi \in S_{h} .
$$

The weak formulation of the problem (1.1), (1.3) is: Find $u \in \mathcal{H}_{0}^{1}(\Omega)$ such that

$$
\begin{aligned}
& \left(u_{t}, v\right)+A(u, v)+\int_{0}^{t} K(t-s) B(u(s), v) d s=(f, v), \quad v \in H_{0}^{1}, \\
& u(0)=u_{0}
\end{aligned}
$$

where $A(\cdot, \cdot)$ is the bilinear form associated with the operator $A$ on $\mathcal{H}_{0}^{1} \times \mathcal{H}_{0}^{1}$ and $B(\cdot, \cdot)$ is defined by

$B(u, v)=(\alpha(u) \nabla u+\beta(u), \nabla v)+(\gamma(u) \cdot \nabla u+g(u), v), \quad u, v \in \mathcal{W}^{1, \infty} \cap \mathcal{H}_{0}^{1}$.

$(\cdot, \cdot)$ denotes the inner product in $\mathcal{L}^{2}(\Omega)$. We always assume that $A$ is coercive and continuous with coercivity constant $\nu_{0}$ and continuity constant $\nu_{1}$. That is, we have

$$
\begin{aligned}
A(v, v) & \geq \nu_{0}\|v\|_{1}^{2} \quad \forall v \in \mathcal{H}_{0}^{1}, \\
|A(u, v)| & \leq \nu_{1}\|u\|_{1}\|v\|_{1} \quad \forall u, v \in \mathcal{H}_{0}^{1} .
\end{aligned}
$$


In view of the assumptions on the functions $\alpha(u), \beta(u), \gamma(u)$, and $g(u)$, it is easily verified that there exists a positive constant $\mu_{0}$ such that

$$
|B(u, v)| \leq \mu_{0}\|u\|_{1}\|v\|_{1} .
$$

For the time discretization of (1.1)-(1.3) we will consider the backward Euler scheme. To analyze the discretization on a time interval $(0, T]$, let $N$ be a positive integer, $\Delta t=T / N$, and let $t_{n}=n \Delta t$. As the truncation error of the backward Euler scheme is $O(\Delta t)$, we introduce a quadrature formula with a truncation error $O(\Delta t)$,

$$
\Delta t \sum_{i=1}^{n} \omega_{n i} g\left(t_{i}\right)=\int_{0}^{t_{n}} K\left(t_{n}-s\right) g(s) d s+O(\Delta t) .
$$

Given our emphasis on two-grid discretization in space, we will not discuss how to obtain the numbers $\omega_{n i}$, but only assume that there exists a positive constant $K_{1}$ such that $\left|\omega_{n i}\right| \leq K_{1}$ for any $1 \leq n \leq N, 1 \leq i \leq n$ and that $\omega_{n n} \neq 0$. Therefore, the problem considered in this paper must be discretized by a fully implicit scheme. Thus, the backward Euler fully discrete finite element approximation of problem (1.1), (1.3) is defined as a sequence $\left\{U^{n}\right\}_{n=0}^{N}$, such that

$$
\begin{aligned}
& \left(\bar{\partial} U^{n}, v\right)+A\left(U^{n}, v\right)+\Delta t \sum_{i=1}^{n} \omega_{n i} B\left(U^{i}, v\right)=\left(f^{n}, v\right), v \in S_{h}, n \geq(12.9) \\
& U^{0}=u_{0}^{h},
\end{aligned}
$$

where $\bar{\partial} U^{n}=\frac{U^{n}-U^{n-1}}{\Delta t}, u_{0}^{h}$ is an appropriate approximation of $u_{0}$ in $S_{h}$, $f^{n}=f\left(t_{n}\right)$. We know that $(2.9)$ will result in a truncation error $O(\Delta t)$ in time. But for nonlinear problems considered in this paper $\left(\omega_{n n} \neq 0\right)$, the solution of a nonlinear algebraic system is required at each time step. To decrease the amount of computational work, we propose using a two-grid technique to solve the PIDEs with nonlinear memory. With this technique, at each time step, solving a nonlinear problem on the fine space $S_{h}$ is reduced by solving a nonlinear problem on the coarse space $S_{H}$ and solving a linear SPD problem on the fine space $S_{h}$.

For functions that vanish on the boundary, we recall Poincare's inequality: there exists a constant $\mathcal{P}$ such that

$$
\forall v \in \mathcal{H}_{0}^{1}, \quad\|v\| \leq \mathcal{P}|v|_{1} .
$$


We make extensive use of the $\epsilon$-type inequality $2 a b \leq \epsilon a^{2}+b^{2} / \epsilon, \epsilon>0$, and of the inequality $a^{2}+b^{2} \leq(|a|+|b|)^{2}$. The results of this paper are based on the identity

$$
2\left(a^{n+1}, a^{n+1}-a^{n}\right)=\left|a^{n+1}\right|^{2}-\left|a^{n}\right|^{2}+\left|a^{n+1}-a^{n}\right|^{2},
$$

and the following Gronwall lemma proved in [18].

Lemma 2.1 (Discrete Gronwall lemma [18]) Let $0 \leq \lambda<1$, and $a_{n}, b_{n}, c_{n}, \lambda_{n} \geq$ 0 with $\left\{c_{n}\right\}$ being monotonically increasing. Then

$$
a_{n}+b_{n} \leq \sum_{j=\varpi}^{n-1} \lambda_{j} a_{j}+\lambda a_{n}+c_{n}, \quad n=\varpi, \varpi+1, \cdots
$$

implies for $n=\varpi, \varpi+1, \cdots$

$$
a_{n}+b_{n} \leq \frac{c_{n}}{1-\lambda} \prod_{j=\varpi}^{n-1}\left(1+\frac{\lambda_{j}}{1-\lambda}\right) \leq \frac{c_{n}}{1-\lambda} \exp \left(\frac{1}{1-\lambda} \sum_{j=\varpi}^{n-1} \lambda_{j}\right) .
$$

\section{Error estimate for the classic fully discrete finite element method}

In this section, we discuss the stability and error estimate of the standard fully discrete finite element method (2.9), (2.10). First, we prove the stability of the solution of (2.9) and (2.10).

Theorem 3.1 Let $U^{n}$ be the solution obtained by (2.9) and (2.10). Then for all

$$
\Delta t \leq \min \left\{\frac{1}{2}, \frac{7 \nu_{0}^{2}}{8 \mu_{0}^{2} K_{1}^{2} T}\right\}
$$

we have

$$
\begin{aligned}
& \left\|U^{n}\right\|+\left(\sum_{i=1}^{n}\left\|U^{i}-U^{i-1}\right\|^{2}\right)^{1 / 2}+\frac{\sqrt{\nu_{0}}}{2}\left(\sum_{i=1}^{n} \Delta t\left\|U^{i}\right\|_{1}^{2}\right)^{1 / 2} \\
\leq & E_{n}^{1 / 2}\left(\left\|U^{0}\right\|^{2}+\Delta t \sum_{i=1}^{n}\left\|f^{i}\right\|^{2}\right)^{1 / 2},
\end{aligned}
$$

where $E_{n}=6 \max \left\{e^{2 t_{n}}, e^{\left(2 \mu_{0} K_{1} t_{n} / \nu_{0}\right)^{2}}\right\}$. 
Proof. By taking $v=2 \Delta t U^{n}$ in (2.9) and using (2.11), we obtain

$$
\begin{aligned}
& \left\|U^{n}\right\|^{2}-\left\|U^{n-1}\right\|^{2}+\left\|U^{n}-U^{n-1}\right\|^{2}+2 \nu_{0} \Delta t\left\|U^{n}\right\|_{1}^{2}+2(\Delta t)^{2} \sum_{i=1}^{n} \omega_{n i} B\left(U^{i}, U^{n}\right) \\
\leq & 2 \Delta t\left\|f^{n}\right\|\left\|U^{n}\right\| .
\end{aligned}
$$

Using (2.7), we have

$$
\begin{gathered}
\left\|U^{n}\right\|^{2}-\left\|U^{n-1}\right\|^{2}+\left\|U^{n}-U^{n-1}\right\|^{2}+2 \nu_{0} \Delta t\left\|U^{n}\right\|_{1}^{2} \\
\leq \mu_{0}(\Delta t)^{2} \sum_{i=1}^{n}\left|\omega_{n i}\right|\left(\frac{1}{\epsilon}\left\|U^{i}\right\|_{1}^{2}+\epsilon\left\|U^{n}\right\|_{1}^{2}\right)+\Delta t\left(\left\|U^{n}\right\|^{2}+\left\|f^{n}\right\|^{2}\right) .
\end{gathered}
$$

Choose $\epsilon=\nu_{0} /\left(\mu_{0} K_{1} t_{n}\right)$ to obtain

$$
\begin{aligned}
& \left\|U^{n}\right\|^{2}+\left\|U^{n}-U^{n-1}\right\|^{2}+\nu_{0} \Delta t\left\|U^{n}\right\|_{1}^{2} \\
\leq & \left\|U^{n-1}\right\|^{2}+\frac{\mu_{0}^{2} K_{1}^{2} t_{n}}{\nu_{0}}(\Delta t)^{2} \sum_{i=1}^{n}\left\|U^{i}\right\|_{1}^{2}+\Delta t\left\|f^{n}\right\|^{2}+\Delta t\left\|U^{n}\right\|^{2} .
\end{aligned}
$$

By summation, we have

$$
\begin{gathered}
\left\|U^{n}\right\|^{2}+\sum_{i=1}^{n}\left\|U^{i}-U^{i-1}\right\|^{2}+\nu_{0} \Delta t \sum_{i=1}^{n}\left\|U^{i}\right\|_{1}^{2} \\
\leq\left\|U^{0}\right\|^{2}+\Delta t \sum_{i=1}^{n}\left\|U^{i}\right\|^{2}+(\Delta t)^{2} \sum_{i=1}^{n} \frac{\mu_{0}^{2} K_{1}^{2} t_{i}}{\nu_{0}} \sum_{j=1}^{i}\left\|U^{j}\right\|_{1}^{2}+\Delta t \sum_{i=1}^{n} \| f^{i}\left(\beta_{2}^{2}, 6\right)
\end{gathered}
$$

which implies that

$$
\begin{aligned}
& (1-\Delta t)\left\|U^{n}\right\|^{2}+\sum_{i=1}^{n}\left\|U^{i}-U^{i-1}\right\|^{2}+\left(\nu_{0}-\frac{\mu_{0}^{2} K_{1}^{2} t_{n}}{\nu_{0}} \Delta t\right) \Delta t \sum_{i=1}^{n}\left\|U^{i}\right\|_{1}^{2} \\
\leq & \left\|U^{0}\right\|^{2}+\Delta t \sum_{i=1}^{n-1} \max \left\{1, \frac{4 \mu_{0}^{2} K_{1}^{2} t_{i}}{\nu_{0}^{2}}\right\}\left(\left\|U^{i}\right\|^{2}+\frac{\nu_{0}}{4} \Delta t \sum_{j=1}^{i}\left\|U^{j}\right\|_{1}^{2}\right)+\Delta t \sum_{i=1}^{n}\left\|f^{i}\right\|^{2} .
\end{aligned}
$$

Since condition (3.1) implies that $1-\Delta t \geq \frac{1}{2}$ and $\nu_{0}-\frac{\mu_{0}^{2} K_{1}^{2} t_{n}}{\nu_{0}} \Delta t \geq \frac{\nu_{0}}{8}$, with 
the aid of discrete Gronwall lemma 2.1, we obtain

$$
\begin{aligned}
& \left\|U^{n}\right\|^{2}+2 \sum_{i=1}^{n}\left\|U^{i}-U^{i-1}\right\|^{2}+\frac{\Delta t \nu_{0}}{4} \sum_{i=1}^{n}\left\|U^{i}\right\|_{1}^{2} \\
\leq & E_{n}\left(\left\|U^{0}\right\|^{2}+\Delta t \sum_{i=1}^{n}\left\|f^{i}\right\|^{2}\right),
\end{aligned}
$$

which implies (3.2). Thus the proof is completed.

Remark. From (3.1), we find that for a given integral interval $(0, T]$ the stepsize $\Delta t$ is determined by the ratio of $\nu_{0}$ to $\mu_{0}$ and increases as the value of coercivity constant $\nu_{0}$ increases.

Now let us take $v=2 \Delta t \bar{\partial} U^{n}$ in (2.9) to obtain

$$
\begin{aligned}
& 2 \Delta t\left\|\bar{\partial} U^{n}\right\|^{2}+A\left(U^{n}, U^{n}\right)-A\left(U^{n-1}, U^{n-1}\right)+A\left(U^{n}-U^{n-1}, U^{n}-U^{n-1}\right) \\
& +2(\Delta t)^{2} \sum_{i=1}^{n} \omega_{n i} B\left(U^{i}, \bar{\partial} U^{n}\right) \\
= & 2 \Delta t\left(f^{n}, \bar{\partial} U^{n}\right) .
\end{aligned}
$$

Since

$$
2 \Delta t\left(f^{n}, \bar{\partial} U^{n}\right) \leq \frac{1}{2} \Delta t\left\|f^{n}\right\|^{2}+2 \Delta t\left\|\bar{\partial} U^{n}\right\|^{2}
$$

and

$$
\begin{aligned}
2(\Delta t)^{2} \sum_{i=1}^{n}\left|\omega_{n i} B\left(U^{i}, \bar{\partial} U^{n}\right)\right| & \leq 2 \Delta t \mu_{0} \sum_{i=1}^{n}\left|\omega_{n i}\right|\left\|U^{i}\right\|_{1}\left\|U^{n}-U^{n-1}\right\|_{1} \\
& \leq \frac{t_{n} \mu_{0}^{2} K_{1}^{2}}{\nu_{0}} \Delta t \sum_{i=1}^{n}\left\|U^{i}\right\|_{1}^{2}+\nu_{0} \| U^{n}-U^{n-1}(\$|\cdot| 1,1)
\end{aligned}
$$

(3.9) becomes

$$
\nu_{0}\left\|U^{n}\right\|_{1}^{2} \leq \frac{1}{2} \Delta t\left\|f^{n}\right\|^{2}+\frac{t_{n} \mu_{0}^{2} K_{1}^{2}}{\nu_{0}} \Delta t \sum_{i=1}^{n}\left\|U^{i}\right\|_{1}^{2}+\nu_{1}\left\|U^{n-1}\right\|_{1}^{2} .
$$

Then we have the following result. 
Theorem 3.2 Let $U^{n}$ be the solution obtained by (2.9) and (2.10). Then for all

$$
\Delta t \leq \frac{\nu_{0}^{2}}{2 \mu_{0}^{2} K_{1}^{2} T}
$$

we have

$$
\left\|U^{n}\right\|_{1} \leq C\left(\left\|U^{0}\right\|_{1}^{2}+\Delta t \sum_{i=1}^{n}\left\|f^{i}\right\|^{2}\right)^{1 / 2}
$$

Proof. It follows from (3.13) that $\nu_{0}-\frac{\mu_{0}^{2} K_{1}^{2} t_{n}}{\nu_{0}} \Delta t \geq \frac{\nu_{0}}{2}$. Then an application of discrete Gronwall lemma 2.1 to (3.12) leads to

$$
\left\|U^{n}\right\|_{1}^{2} \leq C\left(\left\|U^{0}\right\|_{1}^{2}+\Delta t \sum_{i=1}^{n}\left\|f^{i}\right\|^{2}\right),
$$

which implies (3.14). This completes the proof.

To estimate the error of the fully discrete approximation (2.9), we define, for $w, u, v \in \mathcal{W}^{1, \infty} \cap \mathcal{H}_{0}^{1}(\Omega)$,

$$
B_{1}(w ; u, v)=(\alpha(w) \nabla u, \nabla v)+(\gamma(w) \cdot \nabla u, v) .
$$

Due to the assumptions on $\alpha(u)$ and $\gamma(u)$, there exist a constant $\sigma$ such that

$$
\left|B_{1}(w ; u, v)\right| \leq \sigma\|u\|_{1}\|v\|_{1} .
$$

As usual, we write the error $e^{n}=u\left(t_{n}\right)-U^{n}$ as

$$
e^{n}=u\left(t_{n}\right)-U^{n}=\left(u\left(t_{n}\right)-V_{h} u\left(t_{n}\right)\right)+\left(V_{h} u\left(t_{n}\right)-U^{n}\right)=\rho^{n}+\theta^{n},
$$

where $V_{h} u$ is the Ritz-Volterra projection of the solution $u$ and defined by [9]

$$
A\left(u-V_{h} u, v\right)+\int_{0}^{t} K(t-s) B_{1}\left(u(s) ; u(s)-V_{h} u(s), v\right) d s=0, \quad v \in S_{h}(3 .
$$

For $\rho(t)=u(t)-V_{h} u(t)$, following the line of Cannon and Lin [9], we show that there exists $C_{0}>0$, independent of $h$ and $t$, such that (see, also, [12, 30])

$$
\begin{aligned}
\|\rho(t)\|+h\|\rho(t)\|_{1} & \leq C_{0} h^{r}\left|\left\|u(t)|\||_{r}, \quad t \geq 0\right.\right. \\
\left\|\rho_{t}(t)\right\| & \leq C_{0} h^{r}\left(\|\| u(t)\|\|_{r}+\|\| u_{t}(t)\|\|_{r}\right) \\
\|\rho(t)\|_{\infty} & \leq C_{0} h^{r}|\ln h|\|\| u(t)\|\|_{r, \infty}
\end{aligned}
$$


where

$$
\||| u(t) \mid\|_{r}=\|u(t)\|_{r}+\int_{0}^{t}\|u(\tau)\|_{r} d \tau, \quad\|\| u(t)\|\|_{r, \infty}=\|u(t)\|_{r, \infty}+\int_{0}^{t}\|u(\tau)\|_{r, \infty} d \tau,
$$

and there exists a positive constant $C=C(u)$, independent of $h$, such that

$$
\left\|\nabla V_{h} u\right\|_{\infty}+\left\|\nabla\left(V_{h} u\right)_{t}\right\|_{\infty} \leq C
$$

Now we need to estimate the error $\theta^{n}=V_{h} u\left(t_{n}\right)-U^{n}$.

Theorem 3.3 Let $u$ and $U^{n}$ be the solutions of $(2.3)-(2.4)$ and $(2.9)-(2.10)$, respectively. If

$$
16 \sigma^{2} K_{1}^{2} T<\nu_{0}^{2}
$$

then, for sufficiently small $\Delta t$, we have

$$
\left\|\theta^{n}\right\|+\left\|\theta^{n}-\theta^{n-1}\right\|+\sqrt{\nu_{0} \Delta t}\left\|\theta^{n}\right\|_{1} \lesssim h^{r}+\Delta t .
$$

Proof. Firstly, it follows from (2.3) and (2.9) that

$$
\begin{aligned}
& \left(u_{t}-\bar{\partial} U^{n}, v\right)+A\left(u-U^{n}, v\right) \\
& +\int_{0}^{t_{n}} K(t-s) B(u(s), v) d s-\Delta t \sum_{i=1}^{n} \omega_{n i} B\left(U^{i}, v\right)=0, \quad v \in S_{h} .
\end{aligned}
$$

Then we find that $\theta^{n}$ satisfies

$$
\begin{aligned}
& \left(\bar{\partial} \theta^{n}, v\right)+A\left(\theta^{n}, v\right)+A\left(\rho^{n}, v\right)+\int_{0}^{t_{n}} K(t-s) B_{1}\left(u(s) ; u(s)-V_{h} u(s), v\right) d s \\
& +\int_{0}^{t_{n}} K(t-s) B_{1}\left(u(s) ; V_{h} u(s), v\right) d s \\
& +\int_{0}^{t_{n}} K(t-s)[(\beta(u(s)), \nabla v)+(g(u(s)), v)] d s-\Delta t \sum_{i=1}^{n} \omega_{n i} B\left(U^{i}, v\right) \\
& =-\left(\frac{\rho^{n}-\rho^{n-1}}{\Delta t}, v\right)-\left(u_{t}-\frac{u\left(t_{n}\right)-u\left(t_{n-1}\right)}{\Delta t}, v\right), \quad v \in S_{h} .
\end{aligned}
$$


Using (3.16), we have

$$
\begin{aligned}
& \left(\bar{\partial} \theta^{n}, v\right)+A\left(\theta^{n}, v\right)+\int_{0}^{t_{n}} K(t-s) B_{1}\left(u(s) ; V_{h} u(s), v\right) d s \\
& -\Delta t \sum_{i=1}^{n} \omega_{n i} B_{1}\left(u\left(t_{i}\right) ; V_{h} u\left(t_{i}\right), v\right)+\Delta t \sum_{i=1}^{n} \omega_{n i} B_{1}\left(u\left(t_{i}\right) ; \theta^{i}, v\right) \\
& +\Delta t \sum_{i=1}^{n} \omega_{n i}\left[\left(\left(\alpha\left(u\left(t_{i}\right)\right)-\alpha\left(U^{i}\right)\right) \nabla U^{i}, \nabla v\right)+\left(\left(\gamma\left(u\left(t_{i}\right)\right)-\gamma\left(U^{i}\right)\right) \cdot \nabla U^{i}, v\right)\right] \\
& +\int_{0}^{t_{n}} K(t-s)[(\beta(u(s)), \nabla v)+(g(u(s)), v)] d s \\
& -\Delta t \sum_{i=1}^{n} \omega_{n i}\left[\left(\beta\left(u\left(t_{i}\right)\right), \nabla v\right)+\left(g\left(u\left(t_{i}\right)\right), v\right)\right] \\
& +\Delta t \sum_{i=1}^{n} \omega_{n i}\left[\left(\beta\left(u\left(t_{i}\right)\right)-\beta\left(U^{i}\right), \nabla v\right)+\left(g\left(u\left(t_{i}\right)\right)-g\left(U^{i}\right), v\right)\right] \\
& =\left(\frac{\rho^{n}-\rho^{n-1}}{\Delta t}, v\right)-\left(u_{t}-\frac{u\left(t_{n}\right)-u\left(t_{n-1}\right)}{\Delta t}, v\right), \quad v \in S_{h} .
\end{aligned}
$$

Now, in view of (2.8), we have

$\left|\int_{0}^{t_{n}} K(t-s) B_{1}\left(u(s) ; V_{h} u(s), v\right) d s-\Delta t \sum_{i=1}^{n} \omega_{n i} B_{1}\left(u\left(t_{i}\right) ; V_{h} u\left(t_{i}\right), v\right)\right| \lesssim \Delta t\|v\|_{1}$.

and

$$
\begin{aligned}
& \mid \int_{0}^{t_{n}} K(t-s)[(\beta(u(s)), \nabla v)+(g(u(s)), v)] d s \\
& -\Delta t \sum_{i=1}^{n} \omega_{n i}\left[\left(\beta\left(u\left(t_{i}\right)\right), \nabla v\right)+\left(g\left(u\left(t_{i}\right)\right), v\right)\right] \mid \lesssim \Delta t\|v\|_{1} .
\end{aligned}
$$

Due to (3.15), the fifth term on the left-hand side in (3.24) can be bounded as

$$
\left|\Delta t \sum_{i=1}^{n} \omega_{n i} B_{1}\left(u\left(t_{i}\right) ; \theta^{i}, v\right)\right| \leq \Delta t K_{1} \sum_{i=1}^{n} \sigma\left\|\theta^{i}\right\|_{1}\|v\|_{1}
$$


By virtue of the assumptions on $\alpha(u), \beta(u), \gamma(u)$ and $g(u)$, we know $\alpha, \beta, \gamma$ and $g$ satisfy Lipschitz conditions with Lipschitz constant $C_{L}$, and thus the sixth and ninth terms on the left-hand side in (3.24) are estimated as follows:

$$
\begin{aligned}
& \left|\Delta t \sum_{i=1}^{n} \omega_{n i}\left[\left(\left(\alpha\left(u\left(t_{i}\right)\right)-\alpha\left(U^{i}\right)\right) \nabla U^{i}, \nabla v\right)+\left(\left(\gamma\left(u\left(t_{i}\right)\right)-\gamma\left(U^{i}\right)\right) \cdot \nabla U^{i}, v\right)\right]\right| \\
& \leq\left|\Delta t \sum_{i=1}^{n} \omega_{n i}\left[\left(\left(\alpha\left(u\left(t_{i}\right)\right)-\alpha\left(U^{i}\right)\right) \nabla \theta^{i}, \nabla v\right)+\left(\left(\gamma\left(u\left(t_{i}\right)\right)-\gamma\left(U^{i}\right)\right) \cdot \nabla \theta^{i}, v\right)\right]\right| \\
& \Delta t \sum_{i=1}^{n} \omega_{n i}\left[\left(\left(\alpha\left(u\left(t_{i}\right)\right)-\alpha\left(U^{i}\right)\right) \nabla V_{h} u\left(t_{i}\right), \nabla v\right)\right. \\
& \left.+\left(\left(\gamma\left(u\left(t_{i}\right)\right)-\gamma\left(U^{i}\right)\right) \cdot \nabla V_{h} u\left(t_{i}\right), v\right)\right] \mid \\
& \leq \Delta t K_{1} \sum_{i=1}^{n} \sigma\left\|\theta^{i}\right\|_{1}\|v\|_{1}+C_{L} \Delta t K_{1} \sum_{i=1}^{n}\left\|u\left(t_{i}\right)-U^{i}\right\|\left\|\nabla V_{h} u\left(t_{i}\right)\right\|_{\infty}\|v\|_{1} \\
& \leq \Delta t K_{1} \sum_{i=1}^{n} \sigma\left\|\theta^{i}\right\|_{1}\|v\|_{1}+C C_{L} \Delta t K_{1} \sum_{i=1}^{n}\left(\left\|\rho^{i}\right\|+\left\|\theta^{i}\right\|\right)\|v\|_{1} \text {, }
\end{aligned}
$$

where the estimate (3.20) has been used, and

$$
\begin{aligned}
& \left|\Delta t \sum_{i=1}^{n} \omega_{n i}\left[\left(\beta\left(u\left(t_{i}\right)\right)-\beta\left(U^{i}\right), \nabla v\right)+\left(g\left(u\left(t_{i}\right)\right)-g\left(U^{i}\right), v\right)\right]\right| \\
\leq & C_{L} \Delta t K_{1} \sum_{i=1}^{n}\left\|u\left(t_{i}\right)-U^{i}\right\|\|v\|_{1} \leq C_{L} \Delta t K_{1} \sum_{i=1}^{n}\left(\left\|\rho^{i}\right\|+\left\|\theta^{i}\right\|\right)\|v\|(3.29)
\end{aligned}
$$

The first term on the right-hand side in (3.24) can be bounded as

$$
\begin{aligned}
\left|\left(\frac{\rho^{n}-\rho^{n-1}}{\Delta t}, v\right)\right| & \leq \frac{1}{\Delta t}\left\|\rho^{n}-\rho^{n-1}\right\|\|v\| \leq \frac{1}{\Delta t} \int_{t_{n-1}}^{t_{n}}\left\|\rho_{t}(s)\right\| d s\|v\| \\
& \leq \frac{C_{0} h^{r}}{\Delta t} \int_{t_{n-1}}^{t_{n}}\left(\left\|\left|u(s)\left\|\left.\right|_{r}+\right\| u_{t}(s) \|\right|_{r}\right) d s\|v\| ; \quad\right.
\end{aligned}
$$

and the last term can be bounded as

$$
\left|\left(u_{t}-\frac{u\left(t_{n}\right)-u\left(t_{n-1}\right)}{\Delta t}, v\right)\right| \leq \int_{t_{j-1}}^{t_{j}}\left\|u_{t t}\right\| d s\|v\| .
$$


Taking $v=2 \Delta t \theta^{n}$ and substituting all the above estimates (3.25)-(3.31) into (3.24), we obtain

$$
\begin{aligned}
& \left\|\theta^{n}\right\|^{2}-\left\|\theta^{n-1}\right\|^{2}+\left\|\theta^{n}-\theta^{n-1}\right\|^{2}+2 \nu_{0} \Delta t\left\|\theta^{n}\right\|_{1}^{2} \\
\leq & C \Delta t^{2}\left\|\theta^{n}\right\|_{1}+C\left(\Delta t^{2}+\Delta t h^{r}\right)\left\|\theta^{n}\right\|+4 \sigma \Delta t^{2} K_{1} \sum_{i=1}^{n}\left\|\theta^{i}\right\|_{1}\left\|\theta^{n}\right\|_{1} \\
& +4 C C_{L} \Delta t^{2} K_{1} \sum_{i=1}^{n}\left(\left\|\rho^{i}\right\|+\left\|\theta^{i}\right\|\right)\left\|\theta^{n}\right\|_{1} \\
\leq & C \Delta t^{2}+C \Delta t^{2}\left\|\theta^{n}\right\|_{1}^{2}+C\left(\Delta t+h^{r}\right)^{2}+C \Delta t^{2}\left\|\theta^{n}\right\|^{2}+2 \sigma \Delta t^{2} K_{1} \sum_{i=1}^{n} \frac{1}{\epsilon_{1}}\left\|\theta^{i}\right\|_{1}^{2} \\
& +2 \epsilon_{1} \sigma \Delta t K_{1} t_{n}\left\|\theta^{n}\right\|_{1}^{2}+2 C C_{L} \Delta t K_{1} \sum_{i=1}^{n}\left\|\rho^{i}\right\|^{2}+2 C C_{L} \Delta t^{2} K_{1} t_{n}\left\|\theta^{n}\right\|_{1}^{2} \\
& +\frac{2}{\epsilon_{2}} C C_{L} \Delta t^{2} K_{1} \sum_{i=1}^{n}\left\|\theta^{i}\right\|^{2}+2 \epsilon_{2} C C_{L} \Delta t K_{1} t_{n}\left\|\theta^{n}\right\|_{1}^{2},
\end{aligned}
$$

where we have used the inequality

$$
\begin{aligned}
C \Delta t^{2} \sum_{i=1}^{n} a_{i} b_{n} & =C \sum_{i=1}^{n} \Delta t^{1 / 2} a_{i} \Delta t^{3 / 2} b_{n} \\
& \leq \frac{C}{2} \Delta t \sum_{i=1}^{n} a_{i}^{2}+\frac{C}{2} \Delta t^{3} \sum_{i=1}^{n} b_{n}^{2} \leq \frac{C}{2} \Delta t \sum_{i=1}^{n} a_{i}^{2}+\frac{C}{2} \Delta t^{2} t_{n} b_{n}^{2} .
\end{aligned}
$$

Using the estimate (3.17) for $\rho^{i}$, and taking $\epsilon_{1}=\frac{\nu_{0}}{4 \sigma K_{1} t_{n}}$ and $\epsilon_{2}=\frac{\nu_{0}}{4 C C_{L} K_{1} t_{n}}$, we have

$$
\begin{aligned}
& \left\|\theta^{n}\right\|^{2}-\left\|\theta^{n-1}\right\|^{2}+\left\|\theta^{n}-\theta^{n-1}\right\|^{2}+\nu_{0} \Delta t\left\|\theta^{n}\right\|_{1}^{2} \\
\leq & C\left(\Delta t+h^{r}\right)^{2}+\left(C+\frac{8}{\nu_{0}} C^{2} C_{L}^{2} K_{1}^{2} t_{n}\right) \Delta t^{2}\left\|\theta^{n}\right\|^{2} \\
& +\left(C+\frac{8}{\nu_{0}} \sigma^{2} K_{1}^{2} t_{n}+2 C C_{L} K_{1} t_{n}\right) \Delta t^{2}\left\|\theta^{n}\right\|_{1}^{2}+\frac{8}{\nu_{0}} \sigma^{2} \Delta t^{2} K_{1}^{2} t_{n} \sum_{i=1}^{n-1}\left\|\theta^{i}\right\|_{1}^{2} \\
& +\frac{8}{\nu_{0}} C^{2} C_{L}^{2} \Delta t^{2} K_{1}^{2} t_{n} \sum_{i=1}^{n-1}\left\|\theta^{i}\right\|^{2},
\end{aligned}
$$


Noting the condition (3.21) and taking sufficiently small $\Delta t$ such that

$$
\left(C+\frac{8}{\nu_{0}} \sigma^{2} K_{1}^{2} t_{n}+2 C C_{L} K_{1} t_{n}\right) \Delta t \leq \frac{\nu_{0}}{2} \text { and }\left(C+\frac{8}{\nu_{0}} C^{2} C_{L}^{2} K_{1}^{2} t_{n}\right) \Delta t \leq \frac{1}{2},
$$

we obtain

$$
\begin{aligned}
& \left\|\theta^{n}\right\|^{2}+\left\|\theta^{n}-\theta^{n-1}\right\|^{2}+\nu_{0} \Delta t\left\|\theta^{n}\right\|_{1}^{2} \\
\leq & \left\|\theta^{n-1}\right\|^{2}+\frac{1}{2} \Delta t^{2} \sum_{i=1}^{n-1}\left\|\theta^{i}\right\|^{2}+\frac{1}{2} \Delta t\left\|\theta^{n}\right\|^{2}+C\left(\Delta t+h^{r}\right)^{2} \\
& +\frac{\nu_{0}}{2} \Delta t\left\|\theta^{n}\right\|_{1}^{2}+\frac{\nu_{0}}{2} \Delta t^{2} \sum_{i=1}^{n-1}\left\|\theta^{i}\right\|_{1}^{2} .
\end{aligned}
$$

Applying discrete Gronwall lemma 2.1 to the above inequality yields

$$
\left\|\theta^{n}\right\|^{2}+\left\|\theta^{n}-\theta^{n-1}\right\|^{2}+\nu_{0} \Delta t\left\|\theta^{n}\right\|_{1}^{2} \lesssim\left(h^{r}+\Delta t\right)^{2} .
$$

which implies (3.22). This proves the theorem.

Note that the condition (3.21), which implies that the equation (1.1) is diffusion-dominant, is appropriate, since the system may be blowup if the integral term is dominant. Under the condition (3.21), we can not study the long time behaviour of the numerical solution. Of course, if we assume that there exist positive constants $\alpha_{0}, \alpha_{1}>0$ such that

$$
\alpha_{0}|\xi|^{2} \leq \xi^{T} \alpha(u) \xi \leq \alpha_{1}|\xi|^{2}, \quad \forall u \in \mathbb{R}, \quad \xi \in \mathbb{R}^{d}
$$

then following the approach of [45], we can study the long time behavior of the exact solution and the numerical solution.

We now give the $\mathcal{H}^{1}$ estimate of the error $\theta^{n}$.

Theorem 3.4 Let $u$ and $U^{n}$ be the solutions of (2.3)-(2.4) and (2.9)-(2.10), respectively. Then, for all $\Delta t$ satisfying

$$
\Delta t<\frac{\nu_{0}^{2}}{16 \sigma^{2} K_{1}^{2} T}
$$

we have

$$
\left\|\theta^{n}\right\|_{1} \lesssim h^{r}+\Delta t
$$


Proof. Taking $v=2 \Delta t \bar{\partial} \theta^{n}$ in (3.24), and estimating every terms in a way similar to Theorem 3.3, we get

$$
\begin{aligned}
& 2 \Delta t\left\|\bar{\partial} \theta^{n}\right\|^{2}+A\left(\theta^{n}, \theta^{n}\right)-A\left(\theta^{n-1}, \theta^{n-1}\right)+A\left(\theta^{n}-\theta^{n-1}, \theta^{n}-\theta^{n-1}\right) \\
\leq & C \Delta t^{2}\left\|\bar{\partial} \theta^{n}\right\|_{1}+C\left(\Delta t^{2}+\Delta t h^{r}\right)\left\|\bar{\partial} \theta^{n}\right\|+4 \sigma \Delta t^{2} K_{1} \sum_{i=1}^{n}\left\|\theta^{i}\right\|_{1}\left\|\bar{\partial} \theta^{n}\right\|_{1} \\
& +4 C C_{L} \Delta t^{2} K_{1} \sum_{i=1}^{n}\left(\left\|\rho^{i}\right\|+\left\|\theta^{i}\right\|\right)\left\|\bar{\partial} \theta^{n}\right\|_{1} \\
\leq & \frac{C^{2} \Delta t^{2}}{\nu_{0}}+\frac{\nu_{0}}{4}\left\|\theta^{n}-\theta^{n-1}\right\|_{1}^{2}+\frac{C^{2} \Delta t}{8}\left(\Delta t+h^{r}\right)^{2}+2 \Delta t\left\|\bar{\partial} \theta^{n}\right\|^{2} \\
& +\frac{16}{\nu_{0}} \sigma^{2} \Delta t K_{1}^{2} t_{n} \sum_{i=1}^{n}\left\|\theta^{i}\right\|_{1}^{2}+\frac{\nu_{0}}{4}\left\|\theta^{n}-\theta^{n-1}\right\|_{1}^{2}+\frac{16}{\nu_{0}} C^{2} C_{L}^{2} \Delta t K_{1}^{2} t_{n} \sum_{i=1}^{n}\left\|\rho^{i}\right\|^{2} \\
& +\frac{\nu_{0}}{4}\left\|\theta^{n}-\theta^{n-1}\right\|_{1}^{2}+\frac{16}{\nu_{0}} C^{2} C_{L}^{2} \Delta t K_{1}^{2} t_{n} \sum_{i=1}^{n}\left\|\theta^{i}\right\|^{2}+\frac{\nu_{0}}{4}\left\|\theta^{n}-\theta^{n-1}\right\|_{1}^{2} \cdot(3.39)
\end{aligned}
$$

Using (2.3), (2.4), (3.17) and (3.35) yields

$$
\nu_{0}\left\|\theta^{n}\right\|_{1}^{2} \leq \nu_{1}\left\|\theta^{n-1}\right\|_{1}^{2}+C\left(\Delta t+h^{r}\right)^{2}+\frac{16}{\nu_{0}} \sigma^{2} \Delta t K_{1}^{2} t_{n} \sum_{i=1}^{n}\left\|\theta^{i}\right\|_{1}^{2}
$$

Then when $\Delta t$ satisfies (3.37), an application of discrete Gronwall lemma 2.1 to the above inequality leads to (3.38). This completes the proof

We observe that if (3.21) holds, then for any $\Delta t<1$, the conclusion (3.38) is valid.

In the next theorem, we will establish the error estimate for the solution computed by the standard fully discrete finite element method (2.9) $-(2.10)$.

Theorem 3.5 (Error estimate for classic FEM) Let $u$ be the solution of (2.3)-(2.4) and $U^{n}$ be the solution of (2.9)-(2.10). Then, for sufficiently small $\Delta t$, we have, for all $n \geq 1$,

$$
\left\|U^{n}-u\left(t_{n}\right)\right\| \lesssim h^{r}+\Delta t, \quad\left\|U^{n}-u\left(t_{n}\right)\right\|_{1} \lesssim h^{r-1}+\Delta t .
$$

Proof.The first inequality is a direct result of THEOREM 3.3 and (3.17). From THEOREM 3.4 and (3.17), we can prove the second inequality in (3.41). 


\section{Two-grid algorithms for PIDEs with nonlinear memory}

In this section, we present three two-grid algorithms of the backward Euler finite element method for PIDEs with nonlinear memory. The basic mechanism in these algorithms is the construction of two regular triangulations of $\Omega$ : a coarse triangulation $\mathcal{T}_{H}$ with mesh size $H$ and a fine one $\mathcal{T}_{h}$ with mesh size $h(h \ll H)$. For practical purposes, $\mathcal{T}_{h}$ is a refinement of $\mathcal{T}_{H}$. The corresponding finite element spaces are $S_{H}$ and $S_{h}$, which will be called coarse and fine space, respectively. To state the algorithms, we define, for $w, u, v \in \mathcal{W}^{1, \infty} \cap \mathcal{H}_{0}^{1}(\Omega)$,

$$
\tilde{B}(w ; u, v)=(\alpha(w) \nabla u+\beta(w), \nabla v)+(\gamma(w) \cdot \nabla u+g(w), v) .
$$

Due to the assumptions on $\alpha(u), \beta(u), \gamma(u)$, and $g(u)$, there exist two constants $\mu_{1}$ and $\mu_{2}$ such that

$$
|\tilde{B}(w ; u, v)| \leq \mu_{1}\|u\|_{1}\|v\|_{1}+\mu_{2}\|w\|\|v\|_{1} .
$$

Let us now present our first two-grid algorithm.

Algorithm 4.1.

Step one (nonlinear problem on coarse grid $\mathcal{T}_{H}$ ): Given $U_{H}^{n-1}$, find $U_{H}^{n} \in$ $S_{H}$ such that

$$
\begin{aligned}
& \frac{1}{\Delta t}\left(U_{H}^{n}-U_{H}^{n-1}, v\right)+A\left(U_{H}^{n}, v\right)+\Delta t \sum_{i=1}^{n} \omega_{n i} \tilde{B}\left(U_{H}^{i} ; U_{H}^{i}, v\right)=\left(f^{n}, v\right), \\
& \quad v \in S_{H}, n \geq 1 \\
& U_{H}^{0}=u_{H}^{0},
\end{aligned}
$$

Step two (linear problem on fine grid $\mathcal{T}_{h}$ ): Given $U_{H}^{n}$, find $U_{h}^{n} \in S_{h}$ such that

$$
\begin{aligned}
& \frac{1}{\Delta t}\left(U_{h}^{n}-U_{h}^{n-1}, v\right)+A\left(U_{h}^{n}, v\right)+\Delta t \sum_{i=1}^{n} \omega_{n i} \tilde{B}\left(U_{H}^{i} ; U_{h}^{i}, v\right)=\left(f^{n}, v\right), \\
& \quad v \in S_{h}, \quad n \geq 1 \\
& U_{h}^{0}=u_{h}^{0} .
\end{aligned}
$$

Firstly, we observe that for the solution of (4.4) and (4.5), our stability result is similar to the solution of (2.9) and (2.10). 
Theorem 4.1 (Stability of two-grid FEM Algorithm 4.1) Let $U_{h}^{n}$ be the solution obtained by Algorithm 4.1. Then when $\Delta t$ satisfies (3.1) and

$$
\Delta t \leq \min \left\{\frac{1}{2}, \frac{3 \nu_{0}^{2}}{2 \mu_{1}^{2} K_{1}^{2} T}\right\},
$$

we have

$$
\begin{aligned}
& \sup _{1 \leq i \leq n}\left\|U_{h}^{i}\right\|+\left(\sum_{i=1}^{n}\left\|U_{h}^{i}-U_{h}^{i-1}\right\|^{2}\right)^{1 / 2}+\frac{\sqrt{\nu_{0}}}{2}\left(\sum_{i=1}^{n} \Delta t\left\|U_{h}^{i}\right\|_{1}^{2}\right)^{1 / 2} \\
& \leq C\left(\left\|U_{h}^{0}\right\|^{2}+\left\|U_{H}^{0}\right\|^{2}+\Delta t \sum_{i=1}^{n}\left\|f^{i}\right\|^{2}\right)^{1 / 2} .
\end{aligned}
$$

Proof. Similar to (3.4), using (4.1), we have

$$
\begin{aligned}
& \left\|U_{h}^{n}\right\|^{2}-\left\|U_{h}^{n-1}\right\|^{2}+\left\|U_{h}^{n}-U_{h}^{n-1}\right\|^{2}+2 \Delta t \nu_{0}\left\|U_{h}^{n}\right\|_{1}^{2} \\
\leq & (\Delta t)^{2} \sum_{i=1}^{n}\left|\omega_{n i}\right|\left(\frac{\mu_{1}}{4 \epsilon_{1}}\left\|U_{h}^{i}\right\|_{1}^{2}+\mu_{1} \epsilon_{1}\left\|U_{h}^{n}\right\|_{1}^{2}+\frac{\mu_{2}}{4 \epsilon_{2}}\left\|U_{H}^{i}\right\|^{2}+\mu_{2} \epsilon_{2}\left\|U_{h}^{n}\right\|_{1}^{2}\right) \\
& +\Delta t\left(\left\|U_{h}^{n}\right\|^{2}+\left\|f^{n}\right\|^{2}\right) .
\end{aligned}
$$

After choosing $\epsilon_{1}=\frac{\nu_{0}}{2 \mu_{1} K_{1} t_{n}}$ and $\epsilon_{2}=\frac{\nu_{0}}{2 \mu_{2} K_{1} t_{n}}$, (4.8) becomes

$$
\begin{aligned}
& \left\|U_{h}^{n}\right\|^{2}+\left\|U_{h}^{n}-U_{h}^{n-1}\right\|^{2}+\Delta t \nu_{0}\left\|U_{h}^{n}\right\|_{1}^{2} \\
\leq & \left\|U_{h}^{n-1}\right\|^{2}+(\Delta t)^{2} \sum_{i=1}^{n}\left(\frac{\mu_{1}^{2} K_{1}^{2} t_{n}}{2 \nu_{0}}\left\|U_{h}^{i}\right\|_{1}^{2}+\frac{\mu_{2}^{2} K_{1}^{2} t_{n}}{2 \nu_{0}}\left\|U_{H}^{i}\right\|^{2}\right)+\Delta t\left\|f^{n}\right\|^{2}+\Delta t\left\|U_{h}^{n}\right\|^{2} .
\end{aligned}
$$

With arguments similar to those in THEOREM 3.1, we obtain

$$
\begin{aligned}
& \left\|U_{h}^{n}\right\|^{2}+\sum_{i=1}^{n}\left\|U_{h}^{i}-U_{h}^{i-1}\right\|^{2}+\frac{\Delta t \nu_{0}}{4} \sum_{i=1}^{n}\left\|U_{h}^{i}\right\|_{1}^{2} \\
\leq & C\left(\left\|U_{h}^{0}\right\|^{2}+\sup _{1 \leq i \leq n}\left\|U_{H}^{i}\right\|^{2}+\Delta t \sum_{i=1}^{n}\left\|f^{i}\right\|^{2}\right) .
\end{aligned}
$$

As $U_{H}^{i}$ satisfies inequality (3.14), we can obtain (4.7).

To establish the error estimate for the solution computed by ALGORITHM 4.1 , we need the following lemmas. 
Lemma 4.2 Let $U^{n}$ and $U_{h}^{n}$ be the solutions obtained by (2.9)-(2.10) and Algorithm 4.1, respectively. If $\Delta t$ satisfies condition

$$
\Delta t<\frac{\nu_{0}^{2}}{8 \mu_{1}^{2} K_{1}^{2} T}
$$

then for any $n \geq 1$, we have

$$
\frac{2}{\sqrt{\nu_{0} \Delta t}}\left\|W_{h}^{n}-W_{h}^{n-1}\right\|+\left\|W_{h}^{n}\right\|_{1} \lesssim H^{r}+h^{r-1}+\Delta t
$$

where $W_{h}^{n}=U_{h}^{n}-U^{n}$.

Proof. It follows from (2.9) and (4.4) that

$$
\frac{1}{\Delta t}\left(W_{h}^{n}-W_{h}^{n-1}, v\right)+A\left(W_{h}^{n}, v\right)+\Delta t \sum_{i=0}^{n} \omega_{n i}\left(\tilde{B}\left(U_{H}^{i} ; U_{h}^{i}, v\right)-\tilde{B}\left(U^{i} ; U^{i}, v\right)\right)=0 .
$$

Now let us bound $\left|\tilde{B}\left(U_{H}^{i} ; U_{h}^{i}, v\right)-\tilde{B}\left(U^{i} ; U^{i}, v\right)\right|$. Firstly, we split $\tilde{B}\left(U_{H}^{i} ; U_{h}^{i}, v\right)-$ $\tilde{B}\left(U^{i} ; U^{i}, v\right)$ as follows:

$$
\begin{aligned}
& \tilde{B}\left(U_{H}^{i} ; U_{h}^{i}, v\right)-\tilde{B}\left(U^{i} ; U^{i}, v\right) \\
= & \left(\alpha\left(U_{H}^{i}\right) \nabla\left(U_{h}^{i}-U^{i}\right), \nabla v\right)+\left(\left(\alpha\left(U_{H}^{i}\right)-\alpha\left(U^{i}\right)\right) \nabla U^{i}, \nabla v\right)+\left(\beta\left(U_{H}^{i}\right)-\beta\left(U^{i}\right), \nabla v\right) \\
& +\left(\gamma\left(U_{H}^{i}\right) \cdot \nabla\left(U_{h}^{i}-U^{i}\right), v\right)+\left(\left(\gamma\left(U_{H}^{i}\right)-\gamma\left(U^{i}\right)\right) \cdot \nabla U^{i}, v\right)+\left(g\left(U_{H}^{i}\right)-g\left(U^{i}\right), v\right) .
\end{aligned}
$$

It follows that

$$
\begin{aligned}
& \left|\left(\alpha\left(U_{H}^{i}\right) \nabla\left(U_{h}^{i}-U^{i}\right), \nabla v\right)\right|+\left|\left(\beta\left(U_{H}^{i}\right)-\beta\left(U^{i}\right), \nabla v\right)\right| \\
& +\left|\left(\gamma\left(U_{H}^{i}\right) \cdot \nabla\left(U_{h}^{i}-U^{i}\right), v\right)\right|+\left|\left(g\left(U_{H}^{i}\right)-g\left(U^{i}\right), v\right)\right| \\
\leq & \mu_{1}\left\|W_{h}^{i}\right\|_{1}\|v\|_{1}+C_{L}\left\|U_{H}^{i}-U^{i}\right\|\|v\|_{1} \\
\leq & \mu_{1}\left\|W_{h}^{i}\right\|_{1}\|v\|_{1}+C_{L}\left(\left\|u\left(t_{i}\right)-U_{H}^{i}\right\|+\left\|u\left(t_{i}\right)-U^{i}\right\|\right)\|v\|_{1} \\
\leq & \mu_{1}\left\|W_{h}^{i}\right\|_{1}\|v\|_{1}+C_{L}\left(H^{r}+h^{r}+\Delta t\right)\|v\|_{1} .
\end{aligned}
$$

On the other hand, due to the assumption on $\alpha$ and $\gamma$, which implies that $\alpha$ and $\gamma$ are bounded and satisfy Lipschitz condition, we have

$$
\begin{aligned}
& \left.\mid\left(\alpha\left(U_{H}^{i}\right)-\alpha\left(U^{i}\right)\right) \nabla U^{i}, \nabla v\right) \mid \\
\leq & \left.\left.\mid\left(\alpha\left(U_{H}^{i}\right)-\alpha\left(U^{i}\right)\right) \nabla\left(U^{i}-u\left(t_{i}\right)\right), \nabla v\right)|+|\left(\alpha\left(U_{H}^{i}\right)-\alpha\left(U^{i}\right)\right) \nabla u\left(t_{i}\right), \nabla v\right) \mid \\
\leq & C\left\|\nabla\left(U^{i}-u\left(t_{i}\right)\right)\right\|\|\nabla v\|+C_{L}\left\|U_{H}^{i}-U^{i}\right\|\left\|\nabla u\left(t_{i}\right)\right\|_{\infty}\|\nabla v\| \\
\leq & C(u)\left(H^{r}+h^{r-1}+\Delta t\right)\|\nabla v\|,
\end{aligned}
$$


and

$$
\begin{aligned}
& \left.\mid\left(\gamma\left(U_{H}^{i}\right)-\gamma\left(U^{i}\right)\right) \cdot \nabla U^{i}, v\right) \mid \\
\leq & \left.\left.\mid\left(\gamma\left(U_{H}^{i}\right)-\gamma\left(U^{i}\right)\right) \cdot \nabla\left(U^{i}-u\left(t_{i}\right)\right), v\right)|+|\left(\gamma\left(U_{H}^{i}\right)-\gamma\left(U^{i}\right)\right) \cdot \nabla u\left(t_{i}\right), v\right) \mid \\
\leq & C\left\|\nabla\left(U^{i}-u\left(t_{i}\right)\right)\right\|\|v\|+C_{L}\left\|U_{H}^{i}-U^{i}\right\|\left\|\nabla u\left(t_{i}\right)\right\|_{\infty}\|v\| \\
\leq & C(u)\left(H^{r}+h^{r-1}+\Delta t\right)\|v\| .
\end{aligned}
$$

Take $v=2\left(W_{h}^{n}-W_{h}^{n-1}\right)$ in (4.13), and combine (4.13), (4.14), (4.15), (4.16) and (4.17) to get

$$
\begin{aligned}
& \frac{2}{\Delta t}\left\|W_{h}^{n}-W_{h}^{n-1}\right\|^{2}+\nu_{0}\left\|W_{h}^{n}\right\|_{1}^{2}+\nu_{0}\left\|W_{h}^{n}-W_{h}^{n-1}\right\|_{1}^{2} \\
\leq & 2 \Delta t \sum_{i=1}^{n}\left|\omega_{n i}\right|\left(\mu_{1}\left\|W_{h}^{i}\right\|_{1}+C\left(H^{r}+h^{r-1}+\Delta t\right)\right)\left\|W_{h}^{n}-W_{h}^{n-1}\right\|_{1}+\nu_{1}\left\|W_{h}^{n-1}\right\|_{1}^{2} .
\end{aligned}
$$

The first term on the right-hand side of the above inequality can be bounded as

$$
\begin{aligned}
& 2 \Delta t \sum_{i=1}^{n}\left|\omega_{n i}\right|\left(\mu_{1}\left\|W_{h}^{i}\right\|_{1}+C\left(H^{r}+h^{r-1}+\Delta t\right)\right)\left\|W_{h}^{n}-W_{h}^{n-1}\right\|_{1} \\
\leq & \frac{4}{\nu_{0}} \Delta t K_{1}^{2} \mu_{1}^{2} t_{n} \sum_{i=1}^{n}\left\|W_{h}^{i}\right\|_{1}^{2}+\frac{4}{\nu_{0}} C^{2} K_{1}^{2} t_{n}^{2}\left(H^{r}+h^{r-1}+\Delta t\right)^{2} \\
& +\frac{\nu_{0}}{2}\left\|W_{h}^{n}-W_{h}^{n-1}\right\|_{1}^{2},
\end{aligned}
$$

where we have used

$$
2 \Delta t \sum_{i=1}^{n} a_{i} b \leq \frac{4 t_{n}}{\nu_{0}} \Delta t \sum_{i=1}^{n} a_{i}^{2}+\frac{\nu_{0}}{4 t_{n}} \Delta t \sum_{i=1}^{n} b^{2}=\frac{4 t_{n}}{\nu_{0}} \Delta t \sum_{i=1}^{n} a_{i}^{2}+\frac{\nu_{0}}{4} b^{2} .
$$

Substituting (4.19) into (4.18), we get

$$
\begin{aligned}
& \frac{2}{\Delta t}\left\|W_{h}^{n}-W_{h}^{n-1}\right\|^{2}+\frac{\nu_{0}}{2}\left\|W_{h}^{n}\right\|_{1}^{2} \\
\leq & \nu_{1}\left\|W_{h}^{n-1}\right\|_{1}^{2}+\frac{4 \mu_{1}^{2} K_{1}^{2} t_{n}}{\nu_{0}} \Delta t \sum_{i=1}^{n}\left\|W_{h}^{i}\right\|_{1}+C\left(H^{r}+h^{r-1}+\Delta t\right)^{2}(.4
\end{aligned}
$$


In view of (4.11), application of discrete Gronwall lemma 2.1 to the above inequality yields

$$
\frac{4}{\nu_{0} \Delta t}\left\|W_{h}^{n}-W_{h}^{n-1}\right\|^{2}+\left\|W_{h}^{n}\right\|_{1}^{2} \leq C\left(H^{r}+h^{r-1}+\Delta t\right)^{2} .
$$

Then we arrive at (4.12).

Combining Theorem 3.5 and Lemma 4.2 immediately yields the following theorem.

Theorem 4.3 (Error estimate for two-grid FEM Algorithm 4.1) Let $u$ be the solution of (2.3) $-(2.4)$ and $U_{h}^{n}$ be the solution of Algorithm 4.1. Then, for sufficiently small $\Delta t$, we have, for all $n \geq 1$,

$$
\left\|U_{h}^{n}-u\left(t_{n}\right)\right\|_{1} \lesssim H^{r}+h^{r-1}+\Delta t
$$

Proof. Using the triangular inequality $\left\|U_{h}^{n}-u\left(t_{n}\right)\right\|_{1} \leq\left\|U^{n}-u\left(t_{n}\right)\right\|_{1}+$ $\left\|U_{h}^{n}-U^{n}\right\|_{1}$, the second inequality in (3.41), and (4.12), we can obtain (4.22).

From (4.22), it is easy to find that when the mesh sizes satisfy $H=$ $O\left(h^{\frac{r-1}{r}}\right)$ the two-grid Algorithm 4.1 achieves the same approximation for PIDEs with nonlinear memory as the classic finite element method does.

Next we will present an algorithm that reduces a nonlinear problem to a symmetric positive definite (SPD) linear problem and a nonlinear system of smaller size.

Algorithm 4.2 .

Step one (nonlinear problem on coarse grid $\mathcal{T}_{H}$ ): Given $U_{H}^{n-1}$, find $U_{H}^{n} \in$ $S_{H}$ such that

$$
\begin{aligned}
& \frac{1}{\Delta t}\left(U_{H}^{n}-U_{H}^{n-1}, v\right)+A\left(U_{H}^{n}, v\right)+\Delta t \sum_{i=1}^{n} \omega_{n i} \tilde{B}\left(U_{H}^{i} ; U_{H}^{i}, v\right)=\left(f^{n}, v\right), \\
& \quad v \in S_{H}, n=1,2, \cdots, \\
& U_{H}^{0}=u_{H}^{0} .
\end{aligned}
$$

Step two (SPD linear problem on fine grid $\mathcal{T}_{h}$ ): Given $U_{H}^{n}$, find $U_{h}^{n} \in S_{h}$ such that

$$
\begin{aligned}
& \frac{1}{\Delta t}\left(U_{h}^{n}-U_{h}^{n-1}, v\right)+A\left(U_{h}^{n}, v\right)+\Delta t \sum_{i=1}^{n-1} \omega_{n i} \tilde{B}\left(U_{h}^{i} ; U_{h}^{i}, v\right)+\Delta t \omega_{n n} \tilde{B}\left(U_{H}^{n} ; U_{H}^{n}, v\right) \\
& \quad=\left(f^{n}, v\right), \quad v \in S_{h}, \\
& U_{h}^{0}=u_{h}^{0}, \quad n=1,2, \cdots .
\end{aligned}
$$


Obviously, this algorithm can also be applied to the nonsymmetric linear problem.

Theorem 4.4 (Stability of two-grid FEM Algorithm 4.2) Let $U_{h}^{n}$ be the solution obtained by Algorithm 4.2. If $\Delta t$ satisfies (3.1), then we have

$$
\begin{aligned}
& \left\|U_{h}^{n}\right\|+\left(\sum_{i=1}^{n}\left\|U_{h}^{i}-U_{h}^{i-1}\right\|^{2}\right)^{1 / 2}+\frac{\sqrt{\nu_{0}}}{2}\left(\sum_{i=1}^{n} \Delta t\left\|U_{h}^{i}\right\|_{1}^{2}\right)^{1 / 2} \\
\leq & C\left(\left\|U_{h}^{0}\right\|^{2}+\Delta t\left\|U_{H}^{0}\right\|_{1}^{2}+\Delta t \sum_{i=1}^{n}\left\|f^{i}\right\|^{2}\right)^{1 / 2} .
\end{aligned}
$$

for any $n \geq 1$.

Proof. Similar to (3.4), using (4.1), we have

$$
\begin{aligned}
& \left\|U_{h}^{n}\right\|^{2}-\left\|U_{h}^{n-1}\right\|^{2}+\left\|U_{h}^{n}-U_{h}^{n-1}\right\|^{2}+2 \Delta t \nu_{0}\left\|U_{h}^{n}\right\|_{1}^{2} \\
\leq & (\Delta t)^{2} \sum_{i=1}^{n-1}\left|\omega_{n i}\right|\left(\frac{\mu_{0}}{\epsilon}\left\|U_{h}^{i}\right\|_{1}^{2}+\mu_{0} \epsilon\left\|U_{h}^{n}\right\|_{1}^{2}\right)+(\Delta t)^{2}\left|\omega_{n n}\right|\left(\frac{\mu_{0}}{\epsilon}\left\|U_{H}^{n}\right\|_{1}^{2}+\mu_{0} \epsilon\left\|U_{h}^{n}\right\|_{1}^{2}\right) \\
& +\Delta t\left(\left\|U_{h}^{n}\right\|^{2}+\left\|f^{n}\right\|^{2}\right) .
\end{aligned}
$$

After choosing $\epsilon=\frac{\nu_{0}}{\mu_{0} K_{1} t_{n}}$, the above inequality becomes

$$
\begin{aligned}
& \left\|U_{h}^{n}\right\|^{2}+\left\|U_{h}^{n}-U_{h}^{n-1}\right\|^{2}+\Delta t \nu_{0}\left\|U_{h}^{n}\right\|_{1}^{2} \\
\leq & \left\|U_{h}^{n-1}\right\|^{2}+(\Delta t)^{2}\left(\sum_{i=1}^{n-1} \frac{\mu_{0}^{2} K_{1}^{2} t_{n}}{\nu_{0}}\left\|U_{h}^{i}\right\|_{1}^{2}+\frac{\mu_{0}^{2} K_{1}^{2} t_{n}}{\nu_{0}}\left\|U_{H}^{n}\right\|_{1}^{2}\right)+\Delta t\left\|f^{n}\right\|^{2}+\Delta t\left\|U_{h}^{n}\right\|^{2} .
\end{aligned}
$$

With arguments similar to those in THEOREM 3.1, we obtain

$$
\begin{gathered}
\left\|U_{h}^{n}\right\|^{2}+\sum_{i=1}^{n}\left\|U_{h}^{i}-U_{h}^{i-1}\right\|^{2}+\frac{\Delta t \nu_{0}}{4} \sum_{i=1}^{n}\left\|U_{h}^{i}\right\|_{1}^{2} \\
\leq C\left(\left\|U_{h}^{0}\right\|^{2}+(\Delta t)^{2} \sum_{i=1}^{n}\left\|U_{H}^{i}\right\|_{1}^{2}+\Delta t \sum_{i=1}^{n}\left\|f^{i}\right\|^{2}\right),
\end{gathered}
$$


in view of (3.14), therefore,

$$
\begin{aligned}
& \left\|U_{h}^{n}\right\|^{2}+\sum_{i=1}^{n}\left\|U_{h}^{i}-U_{h}^{i-1}\right\|^{2}+\frac{\Delta t \nu_{0}}{4} \sum_{i=1}^{n}\left\|U_{h}^{i}\right\|_{1}^{2} \\
\leq & C\left(\left\|U_{h}^{0}\right\|^{2}+\Delta t\left\|U_{H}^{0}\right\|_{1}^{2}+\Delta t \sum_{i=1}^{n}\left\|f^{i}\right\|^{2}\right),
\end{aligned}
$$

which implies (4.27). This completes the proof.

Theorem 4.5 (Error estimate for two-grid FEM Algorithm 4.2) Let $U_{h}^{n}$ be the solution obtained by Algorithm 4.2. Then for sufficient small $\Delta t$, we have

$$
\left\|U_{h}^{n}-u\left(t_{n}\right)\right\| \lesssim \sqrt{\Delta t} H^{r-1}+h^{r}+\Delta t
$$

for any $n \geq 1$.

Proof. As in Theorem 4.4, $W_{h}^{n}=U_{h}^{n}-U^{n}$ satisfies the following error equation:

$$
\begin{aligned}
& \frac{1}{\Delta t}\left(W_{h}^{n}-W_{h}^{n-1}, v\right)+A\left(W_{h}^{n}, v\right)+\Delta t \sum_{i=0}^{n-1} \omega_{n i}\left(\tilde{B}\left(U_{h}^{i} ; U_{h}^{i}, v\right)-\tilde{B}\left(U^{i} ; U^{i}, v\right)\right) \\
& +\Delta t \omega_{n n}\left(\tilde{B}\left(U_{H}^{n} ; U_{H}^{n}, v\right)-\tilde{B}\left(U^{n} ; U^{n}, v\right)\right)=0
\end{aligned}
$$

In view of the assumption on the coefficients of $B$, there exists a constants $\mu_{B}$ such that

$$
|\tilde{B}(u ; u, v)-\tilde{B}(w ; w, v)| \leq \mu_{B}\|u-w\|_{1}\|v\|_{1} .
$$

Then we have

$$
\begin{aligned}
& \left|\Delta t \omega_{n n}\left(\tilde{B}\left(U_{H}^{n} ; U_{H}^{n}, v\right)-\tilde{B}\left(U^{n} ; U^{n}, v\right)\right)\right| \\
\leq & \mu_{B} \Delta t\left|\omega_{n n}\right|\left\|U_{H}^{n}-U^{n}\right\|_{1}\|v\|_{1} \\
\leq & \mu_{B} K_{1} \Delta t\left(\left\|U_{H}^{n}-u\left(t_{n}\right)\right\|_{1}+\left\|u\left(t_{n}\right)-U^{n}\right\|_{1}\right)\|v\|_{1} \\
\leq & \mu_{B} K_{1} \Delta t\left(H^{r-1}+h^{r-1}+\Delta t\right)\|v\|_{1} .
\end{aligned}
$$


Take $v=2 \Delta t W_{h}^{n}$ in (4.32) to obtain

$$
\begin{aligned}
& \left\|W_{h}^{n}\right\|^{2}-\left\|W_{h}^{n-1}\right\|^{2}+\left\|W_{h}^{n}-W_{h}^{n-1}\right\|^{2}+2 \Delta t \nu_{0}\left\|W_{h}^{n}\right\|_{1}^{2} \\
\leq & \mu_{B}(\Delta t)^{2} \sum_{i=1}^{n-1}\left|\omega_{n i}\right|\left(\frac{1}{\epsilon}\left\|W_{h}^{i}\right\|_{1}^{2}+\epsilon\left\|W_{h}^{n}\right\|_{1}^{2}\right) \\
& +\mu_{B} K_{1} \Delta t\left(\epsilon \Delta t\left\|W_{h}^{n}\right\|_{1}^{2}+\frac{1}{\epsilon} \Delta t\left(H^{r-1}+\Delta t\right)^{2}\right) .
\end{aligned}
$$

By choosing $\epsilon=\frac{\nu_{0}}{t_{n} \mu_{B} K_{1}}$, we get

$$
\begin{gathered}
\left\|W_{h}^{n}\right\|^{2}-\left\|W_{h}^{n-1}\right\|^{2}+\left\|W_{h}^{n}-W_{h}^{n-1}\right\|^{2}+\Delta t \nu_{0}\left\|W_{h}^{n}\right\|_{1}^{2} \\
\leq \frac{t_{n} \mu_{B}^{2} K_{1}^{2}}{\nu_{0}}(\Delta t)^{2} \sum_{i=1}^{n-1}\left\|W_{h}^{i}\right\|_{1}^{2}+\frac{t_{n} \mu_{B}^{2} K_{1}^{2}}{\nu_{0}}(\Delta t)^{2}\left(H^{r-1}+\Delta t\right)^{2} .
\end{gathered}
$$

Sum from 1 up to $n$ to obtain

$$
\begin{aligned}
& \left\|W_{h}^{n}\right\|^{2}-\left\|W_{h}^{0}\right\|^{2}+\sum_{i=1}^{n}\left\|W_{h}^{i}-W_{h}^{i-1}\right\|^{2}+\Delta t \nu_{0} \sum_{i=1}^{n}\left\|W_{h}^{i}\right\|_{1}^{2} \\
\leq & (\Delta t)^{2} \sum_{i=1}^{n} \frac{t_{i} \mu_{B}^{2} K_{1}^{2}}{\nu_{0}} \sum_{j=1}^{i-1}\left\|W_{h}^{j}\right\|_{1}^{2}+\sum_{i=1}^{n} \frac{t_{i} \mu_{B}^{2} K_{1}^{2}}{\nu_{0}}(\Delta t)^{2}\left(H^{r-1}+\Delta t\right)^{2} \\
\leq & (\Delta t)^{2} \sum_{i=0}^{n-1} \frac{t_{i+1} \mu_{B}^{2} K_{1}^{2}}{\nu_{0}} \sum_{j=1}^{i}\left\|W_{h}^{j}\right\|_{1}^{2}+\sum_{i=1}^{n} \frac{t_{i} \mu_{B}^{2} K_{1}^{2}}{\nu_{0}}(\Delta t)^{2}\left(H^{r-1}+\Delta t\right)^{2} \\
\leq & (\Delta t)^{2} \sum_{i=0}^{n-1} \frac{t_{i+1} \mu_{B}^{2} K_{1}^{2}}{\nu_{0}} \sum_{j=1}^{i}\left\|W_{h}^{j}\right\|_{1}^{2}+\frac{t_{n}^{2} \mu_{B}^{2} K_{1}^{2}}{\nu_{0}} \Delta t\left(H^{r-1}+\Delta t\right)^{2} .
\end{aligned}
$$

An application of discrete Gronwall Lemma 2.1 yields

$$
\begin{aligned}
& \left\|W_{h}^{n}\right\|^{2}+\sum_{i=1}^{n}\left\|W_{h}^{i}-W_{h}^{i-1}\right\|^{2}+\Delta t \nu_{0} \sum_{i=1}^{n}\left\|W_{h}^{n}\right\|_{1}^{2} \\
\leq & \frac{t_{n}^{2} \mu_{B}^{2} K_{1}^{2}}{\nu_{0}} \Delta t\left(H^{r-1}+\Delta t\right)^{2} \exp \left(\frac{t_{n}^{2} \mu_{B}^{2} K_{1}^{2}}{\nu_{0}^{2}}\right) .
\end{aligned}
$$

Finally, (4.31) follows readily from this result when a triangular inequality is also applied. 
Next we will present an algorithm that significantly reduces computational memory and storage requirements when $B$ gathers lower-order spatial derivatives and nonlinear terms. To state the algorithm, we define

$$
\tilde{B}_{s}(w ; u, v)=(\alpha(w) \nabla u, \nabla v),
$$

and

$$
N(w ; u, v)=(\beta(w), \nabla v)+(\gamma(w) \cdot \nabla u+g(w), v) .
$$

In view of the assumptions on $\alpha(u), \beta(u), \gamma(u)$, and $g(u)$, we find that there exist two constants $\mu_{3}$ and $\mu_{4}$ such that

$$
\begin{aligned}
\left|\tilde{B}_{s}(w ; u, v)\right| & \leq \mu_{3}\|u\|_{1}\|v\|_{1} \\
|N(w ; u, v)| & \leq \mu_{4}\|u\|\|v\|_{1} .
\end{aligned}
$$

Then the algorithm can be stated as follows.

Algorithm 4.3.

Step one (nonlinear problem on coarse grid $\mathcal{T}_{H}$ ): Given $U_{H}^{n-1}$, find $U_{H}^{n} \in$ $S_{H}$ such that

$$
\begin{aligned}
& \frac{1}{\Delta t}\left(U_{H}^{n}-U_{H}^{n-1}, v\right)+A\left(U_{H}^{n}, v\right)+\Delta t \sum_{i=1}^{n} \omega_{n i} \tilde{B}\left(U_{H}^{i} ; U_{H}^{i}, v\right)=\left(f^{n}, v\right), \\
& \quad v \in S_{H}, n \geq 1 \\
& U_{H}^{0}=u_{H}^{0} .
\end{aligned}
$$

Step two (linear problem on fine grid $\mathcal{T}_{h}$ ): Given $U_{H}^{n}$, find $U_{h}^{n} \in S_{h}$ such that

$$
\begin{aligned}
& \frac{1}{\Delta t}\left(U_{h}^{n}-U_{h}^{n-1}, v\right)+A\left(U_{h}^{n}, v\right)+\Delta t \sum_{i=1}^{n} \omega_{n i}\left(\tilde{B}_{s}\left(U_{H}^{i} ; U_{h}^{i}, v\right)+N\left(U_{H}^{i} ; U_{H}^{i}, v\right)\right) \\
& \quad=\left(f^{n}, v\right), \quad v \in S_{h}, \quad n \geq 1, \\
& U_{h}^{0}=u_{h}^{0},
\end{aligned}
$$

The stability of ALGORITHM 4.3 can be obtained by the same argument for THEOREM 4.1.

Theorem 4.6 (Stability of two-grid FEM Algorithm 4.3) Let $U_{h}^{n}$ be the solution obtained by Algorithm 4.3. Then when

$$
\Delta t \leq \min \left\{\frac{1}{2}, \frac{3 \nu_{0}^{2}}{2 \mu_{3}^{2} K_{1}^{2} T}\right\},
$$


we have

$$
\begin{aligned}
& \sup _{1 \leq i \leq n}\left\|U_{h}^{i}\right\|+\left(\sum_{i=1}^{n}\left\|U_{h}^{i}-U_{h}^{i-1}\right\|^{2}\right)^{1 / 2}+\frac{\sqrt{\nu_{0}}}{2}\left(\sum_{i=1}^{n} \Delta t\left\|U_{h}^{i}\right\|_{1}^{2}\right)^{1 / 2} \\
\leq & C\left(\left\|U_{h}^{0}\right\|^{2}+\left\|U_{H}^{0}\right\|^{2}+\Delta t \sum_{i=1}^{n}\left\|f^{i}\right\|^{2}\right)^{1 / 2} .
\end{aligned}
$$

Proof. Similar to (3.4), using (4.38) and (4.39), we have

$$
\begin{aligned}
& \left\|U_{h}^{n}\right\|^{2}-\left\|U_{h}^{n-1}\right\|^{2}+\left\|U_{h}^{n}-U_{h}^{n-1}\right\|^{2}+2 \Delta t \nu_{0}\left\|U_{h}^{n}\right\|_{1}^{2} \\
\leq & (\Delta t)^{2} \sum_{i=1}^{n}\left|\omega_{n i}\right|\left(\frac{\mu_{3}}{4 \epsilon_{1}}\left\|U_{h}^{i}\right\|_{1}^{2}+\mu_{3} \epsilon_{1}\left\|U_{h}^{n}\right\|_{1}^{2}+\frac{\mu_{4}}{4 \epsilon_{2}}\left\|U_{H}^{i}\right\|^{2}+\mu_{4} \epsilon_{2}\left\|U_{h}^{n}\right\|_{1}^{2}\right) \\
& +\Delta t\left(\left\|U_{h}^{n}\right\|^{2}+\left\|f^{n}\right\|^{2}\right) .
\end{aligned}
$$

After choosing $\epsilon_{1}=\frac{\nu_{0}}{2 \mu_{3} K_{1} t_{n}}$ and $\epsilon_{2}=\frac{\nu_{0}}{2 \mu_{4} K_{1} t_{n}}$, (4.46) becomes

$$
\begin{aligned}
& \left\|U_{h}^{n}\right\|^{2}+\left\|U_{h}^{n}-U_{h}^{n-1}\right\|^{2}+\Delta t \nu\left\|U_{h}^{n}\right\|_{1}^{2} \\
\leq & \left\|U_{h}^{n-1}\right\|^{2}+(\Delta t)^{2} \sum_{i=1}^{n}\left(\frac{\mu_{3}^{2} K_{1}^{2} t_{n}}{2 \nu_{0}}\left\|U_{h}^{i}\right\|_{1}^{2}+\frac{\mu_{4}^{2} K_{1}^{2} t_{n}}{2 \nu_{0}}\left\|U_{H}^{i}\right\|^{2}\right)+\Delta t\left\|f^{n}\right\|^{2}+\Delta t\left\|U_{h}^{n}\right\|^{2} .
\end{aligned}
$$

With arguments similar to those in THEOREM 3.1, we obtain

$$
\begin{aligned}
& \left\|U_{h}^{n}\right\|^{2}+\sum_{i=1}^{n}\left\|U_{h}^{i}-U_{h}^{i-1}\right\|^{2}+\frac{\Delta t \nu_{0}}{4} \sum_{i=1}^{n}\left\|U_{h}^{i}\right\|_{1}^{2} \\
\leq & C\left(\left\|U_{h}^{0}\right\|^{2}+\sup _{1 \leq i \leq n}\left\|U_{H}^{i}\right\|^{2}+\Delta t \sum_{i=1}^{n}\left\|f^{i}\right\|^{2}\right) .
\end{aligned}
$$

As $U_{H}^{i}$ satisfies inequality (3.2), we can obtain (4.45).

To get an idea of the accuracy of Algorithm 4.3, we have the following theorem.

Theorem 4.7 (Error estimate for two-grid FEM Algorithm 4.3) Let $U_{h}^{n}$ be the solutions obtained by ALgorithm 4.3. Then for sufficient small $\Delta t$, we have

$$
\left\|U_{h}^{n}-u\left(t_{n}\right)\right\| \lesssim h^{r}+\Delta t+\sqrt{\Delta t} H^{r}, \quad\left\|U_{h}^{n}-u\left(t_{n}\right)\right\|_{1} \lesssim H^{r}+h^{r-1}+\Delta(4.49)
$$

for any $n \geq 1$. 
Proof. Set $W_{h}^{n}=U_{h}^{n}-U^{n}$ to get

$$
\begin{aligned}
& \frac{1}{\Delta t}\left(W_{h}^{n}-W_{h}^{n-1}, v\right)+A\left(W_{h}^{n}, v\right)+\Delta t \sum_{i=1}^{n} \omega_{n i}\left(\tilde{B}_{s}\left(U_{H}^{i} ; U_{h}^{i}, v\right)-\tilde{B}_{s}\left(U^{i} ; U^{i}, v\right)\right) \\
& +\Delta t \sum_{i=1}^{n} \omega_{n i}\left(N\left(U_{H}^{i} ; U_{H}^{i}, v\right)-N\left(U^{i} ; U^{i}, v\right)\right)=0 .
\end{aligned}
$$

Similar to the proof of LEMma 4.2, we have

$$
\begin{aligned}
& N\left(U_{H}^{i} ; U_{H}^{i}, v\right)-N\left(U^{i} ; U^{i}, v\right) \\
= & \left(\beta\left(U_{H}^{i}\right)-\beta\left(U^{i}\right), \nabla v\right)+\left(\gamma\left(U_{H}^{i}\right) \cdot \nabla\left(U_{H}^{i}-U^{i}\right), v\right) \\
+ & \left(\left(\gamma\left(U_{H}^{i}\right)-\gamma\left(U^{i}\right)\right) \cdot \nabla U^{i}, v\right)+\left(g\left(U_{H}^{i}\right)-g\left(U^{i}\right), \nabla v\right)
\end{aligned}
$$

and

$$
\begin{aligned}
\left|\left(\gamma\left(U_{H}^{i}\right) \cdot \nabla\left(U_{H}^{i}-U^{i}\right), v\right)\right| & \leq C\left\|U_{H}^{i}-U^{i}\right\|\|v\|_{1} \\
& \leq C_{L}\left(\left\|u\left(t_{i}\right)-U_{H}^{i}\right\|+\left\|u\left(t_{i}\right)-U^{i}\right\|\right)\|v\|_{1} \\
& \leq C_{L}\left(H^{r}+h^{r}+\Delta t\right)\|v\|_{1} .
\end{aligned}
$$

The desired estimate can then be obtained in a way similar to proofs of Theorem 4.3 and Lemma 4.2 .

Remark. Observe that when $\alpha \equiv 0$, the approximation of the integral term on the fine grid is identical to the approximation of the integral term on the coarse grid. This means that when we solve $U_{h}^{n}$, all $U_{h}^{i}(i<n-1)$ do not need to be stored on a fine grid. It also means that once the approximation of the integral term has been computed on the coarse grid it does not need to be computed on the fine grid. This significantly reduces computational memory and storage requirements. This result is novel and interesting even for linear problem.

\section{Numerical experiments}

In this section, we show some experiments to confirm the effectiveness and theoretical analysis for Algorithm 4.3. We set the domain as $[0,1] \times[0,1]$ and $T=1.00$. Noting that when $\alpha \equiv 0$ in Algorithm 4.3, the algorithm does not need to store $U_{h}^{i}(i<n-1)$, hence in order to confirm the efficiency and advantage of Algorithm 4.3, we set $K(t)=e^{-t}, \alpha(u)=0, \beta(u)=$ 
$(\sin u, 1-\cos u)^{T}, \gamma(u)=(1-\cos u, \sin u)^{T}, g(u)=\sin u$ in (1.1) and we solve the following problem

$$
\begin{aligned}
& u_{t}-\Delta u+\int_{0}^{t} e^{-(t-s)}(-\nabla \cdot \beta(u(s))+\gamma(u(s)) \cdot \nabla u+g(u(s))) d s \\
& =f\left(x_{1}, x_{2} ; t\right) \\
& u\left(x_{1}, x_{2} ; t\right)=0, \quad\left(x_{1}, x_{2} ; t\right) \in \partial \Omega \times(0, T], \\
& u\left(x_{1}, x_{2} ; 0\right)=u_{0}\left(x_{1}, x_{2}\right), \quad\left(x_{1}, x_{2}\right) \in \Omega .
\end{aligned}
$$

We further set $u_{0}\left(x_{1}, x_{2}\right)=x_{1}\left(1-x_{1}\right) x_{2}\left(1-x_{2}\right)$ and

$$
\begin{aligned}
& f\left(x_{1}, x_{2} ; t\right) \\
& =\left(2 x_{1}\left(1-x_{1}\right)-x_{1}\left(1-x_{1}\right) x_{2}\left(1-x_{2}\right)+2 x_{2}\left(1-x_{2}\right)+\left(1-2 x_{1}\right) x_{2}\left(1-x_{2}\right) t\right) e^{-t} \\
& -2\left(1-2 x_{1}\right) x_{2}\left(1-x_{2}\right) e^{-t} \int_{0}^{t} \cos \left(x_{1}\left(1-x_{1}\right) x_{2}\left(1-x_{2}\right) e^{-s}\right) d s \\
& +e^{-t} \int_{0}^{t} e^{s} \sin \left(x_{1}\left(1-x_{1}\right) x_{2}\left(1-x_{2}\right) e^{-s}\right) d s .
\end{aligned}
$$

Then we can verify that $u\left(x_{1}, x_{2} ; t\right)=x_{1}\left(1-x_{1}\right) x_{2}\left(1-x_{2}\right) e^{-t}$ is the true solution. We use linear finite element for the space discretization. The convergence rate and effectiveness of AlgORITHM 4.3 in $H^{1}$ norm given by Theorem 4.7 are confirmed in Table 1 with $h=\frac{1}{2^{l}}, l=2, \cdots, 9 ; H=\frac{1}{2} \sqrt{h}$ and $\Delta t=\frac{1}{2^{l}}, l=1, \cdots, 8$.

\begin{tabular}{|c|c|c|c|c|c|c|}
\hline$H$ & $h$ & $\Delta t$ & $\left\|U_{h}^{T}-u(T)\right\|_{1}$ & $h$ order & $H$ order & $\Delta t$ order \\
\hline $1 / 4$ & $1 / 4$ & $1 / 2$ & $2.17236 \times 10^{-2}$ & -- & -- & -- \\
\hline $1 / 6$ & $1 / 8$ & $1 / 4$ & $1.11164 \times 10^{-2}$ & 0.95 & 1.95 & 0.95 \\
\hline $1 / 8$ & $1 / 16$ & $1 / 8$ & $5.59226 \times 10^{-3}$ & 0.99 & 1.99 & 0.99 \\
\hline $1 / 12$ & $1 / 32$ & $1 / 16$ & $2.80089 \times 10^{-3}$ & 0.99 & 1.99 & 0.99 \\
\hline $1 / 16$ & $1 / 64$ & $1 / 32$ & $1.40136 \times 10^{-3}$ & 1.00 & 2.00 & 1.00 \\
\hline $1 / 23$ & $1 / 128$ & $1 / 64$ & $7.00760 \times 10^{-4}$ & 0.99 & 1.99 & 0.99 \\
\hline $1 / 32$ & $1 / 256$ & $1 / 128$ & $3.50427 \times 10^{-4}$ & 1.00 & 2.00 & 1.00 \\
\hline $1 / 46$ & $1 / 512$ & $1 / 256$ & $1.75207 \times 10^{-4}$ & 1.00 & 2.00 & 1.00 \\
\hline
\end{tabular}

Table 1: Convergence rate and accuracy of ALgORITHM 4.3.

Following the Algorithm 4.3, in the numerical experiments, we do not store $U_{h}^{i}(i<n-1)$ and save a lot of storege. Further, the method is much 
more efficient than the standard fully discrete finite element algorithm since we only need to solve a nonlinear problem with mesh-size $H=\frac{1}{2} \sqrt{h}$ and then solve the linear problem with mesh-size $h$. Using standard fully discrete finite element algorithm to solve the problem (5.53) by solving the nonlinear problem directly with mesh-size $h$ and the convergence rate and error in $H^{1}$ norm are shown in Table 2, Comparing Table 1 and Table 2, we can clearly see that the effectiveness and accuracy of Algorithm 4.3 are the same as standard fully discrete finite element algorithm. The error estimate in $L^{2}$ norm given by Theorem 4.7 can also be confirmed similarly, for simplicity, we omitted listing the tables here.

\begin{tabular}{|c|c|c|c|c|}
\hline$h$ & $\Delta t$ & $\left\|U_{h}^{T}-u(T)\right\|_{1}$ & $h$ order & $\Delta t$ order \\
\hline $1 / 4$ & $1 / 2$ & $2.17183 \times 10^{-2}$ & -- & -- \\
\hline $1 / 8$ & $1 / 4$ & $1.11115 \times 10^{-2}$ & 0.95 & 0.95 \\
\hline $1 / 16$ & $1 / 8$ & $5.58847 \times 10^{-3}$ & 0.99 & 0.99 \\
\hline $1 / 32$ & $1 / 16$ & $2.79844 \times 10^{-3}$ & 1.00 & 1.00 \\
\hline $1 / 64$ & $1 / 32$ & $1.39977 \times 10^{-3}$ & 1.00 & 1.00 \\
\hline $1 / 128$ & $1 / 64$ & $6.99958 \times 10^{-4}$ & 1.00 & 1.00 \\
\hline $1 / 256$ & $1 / 128$ & $3.49990 \times 10^{-4}$ & 1.00 & 1.00 \\
\hline $1 / 512$ & $1 / 256$ & $1.74996 \times 10^{-4}$ & 1.00 & 1.00 \\
\hline
\end{tabular}

Table 2: Error and convergence rate for standard fully discrete finite element algorithm.

\section{Concluding remarks}

We have presented and derived error estimates for several two-grid finite element algorithms for PIDEs with nonlinear memory. With the backward Euler scheme, the two-grid strategy consists of two steps: (1) discretizing the fully nonlinear problem in space on a coarse grid with mesh-size $H$ and time step-size $\Delta t$ and (2) discretizing the linearized problem in space on a fine grid with mesh-size $h$ and the same time step-size as in step (1). It is shown that these algorithms are as stable as the standard fully discrete finite element algorithm. We also present the error estimate at each time step. Compared with standard finite element methods, our algorithm not only keep good accuracy but also saves a lot of computational cost. As a byproduct of these results, we found that one of these algorithms, Algorithm 4.3, significantly reduces computational memory and storage requirements if the 
nonlinear memory is defined by a first-order or zero-order nonlinear differential operator. Thus, the two-grid methods studied in this paper provide a new approach that takes advantage of some of the nice properties hidden in a complex problem.

Numerical experiments for Algorithm 4.3 are provided to confirm the theoretical results and show that the two-grid method has the same effectiveness and accuracy as the standard fully discrete finite element algorithm.

The analysis herein was carried out for an implicit Euler discretization in time. However, the results could be extended to the second-order accuracy backward differentiation formula (BDF) scheme. Moreover, the analysis is valid for a state-dependent forcing term $f$ that satisfies certain conditions, e.g.,

$$
\left|\frac{\partial}{\partial u} f(x, t, u)\right|+\left|\frac{\partial^{2}}{\partial u^{2}} f(x, t, u)\right| \leq M, \quad u \in \mathbb{R},
$$

where $M$ is a positive constant.

\section{Acknowledgments}

The first author thanks Professor Jinchao Xu for suggesting this problem and for many stimulating and inspiring discussions. This paper was written at the School of Mathematical Sciences, Peking University, where the first author spent time as a visiting scholar.

This work was partially supported by the National Natural Science Foundation of China [grant numers 11771060,11371074].

\section{References}

[1] H. Abboud and T. Sayah, A full discretization of the time-dependent Navier-Stokes equations by a two-grid scheme, M2AN Math. Model. Numer. Anal., 42 (2008), 141-174.

[2] H. Abboud, V. Girault and T. Sayah, A second order accuracy for a fully discretized time-dependent Navier-Stokes equations by a two-grid scheme, Numer. Math., 114 (2009), 189-231.

[3] O. Axelsson and W. Layton, A two-level method for the discretization of nonlinear boundary value problems, SIAM J. Numer. Anal. 33 (1996), 2359-2374. 
[4] C. Bi And V. Ginting, Two-grid finite volume element method for linear and nonlinear elliptic problems, Numer. Math., 108 (2007) 177198.

[5] C. Bi And V. Ginting, Two-grid discontinuous Galerkin method for quasi-linear elliptic problems, J. Sci. Comput., DOI: 10.1007/s10915011-9463-9.

[6] I. H. Biswas, E. R. Jakobsen, and K. H. Karlsen, Differencequadrature schemes for nonlinear degenerate parabolic integro-PDE, SIAM J. Numer. Anal. 48 (2010), 1110-1135.

[7] M. CAI, M. MU AND J. XU, Numerical solution to a mixed NavierStokes/Darcy model by the two-grid approach, SIAM J. Numer. Anal., 47 (2009), 3325-3338.

[8] J. R. Cannon And Y. Lin, Non-classical $H^{1}$ projection and Galerkin methods for nonlinear parabolic integro-differential equation, Calcolo 25 (1988) 187-201.

[9] J. R. CAnnon And Y. Lin, A priori $L^{2}$ error estimates for finite element methods for nonlinear diffusion equations with memory, SIAM J. Numer. Anal. 27 (1990) 595-607.

[10] C. Chen And W. LiU, Two-grid finite volume element methods for semilinear parabolic problems, Appl. Numer. Math., 60 (2010), 10-18.

[11] C. Chen And W. LiU, A two-grid method for finite volume element approximations of second-order nonlinear hyperbolic equations, J. Comput. Appl. Math., 233 (2010), 2975-2984.

[12] C. Chen And T. Shin, Finite element methods for integro-differential equations, Singapore: World Scientifi Pub. Co., 1998.

[13] Y. CHEN AND L. LI, $L^{p}$ error estimates of two-grid schemes of expanded mixed finite element methods, Appl. Math. Comput., 209 (2009), 197205.

[14] Y. Chen, Y. Huang And D. YU, A two-grid method for expanded mixed finite-element solution of semilinear reaction-diffusion equations, Int. J. Numer. Methods Eng. 57 (2003), 193-209. 
[15] X. Dai And X. Cheng, A two-grid method based on Newton iteration for the Navier-Stokes equtions, J. Comput. Appl. Math., 220 (2008), 566-573.

[16] C. N. Dawson And M. F. WheEler, Two-grid method for mixed finite difference approximations fo non-linear parabolic equations, Contemp. Math., 180 (1994), 191-203.

[17] C. N. Dawson, M. F. Wheeler and C. S. Woodward, A twogrid finite difference scheme for nonlinear parabolic equations, SIAM J. Numer. Anal., 35 (1998), 435-452

[18] E. EmmRICH, Stability and error of the variable two-step BDF for semilinear parabolic problems, J. Appl. Math. Computing, 19 (2005), 33-55.

[19] R. E. Ewing, Y. Lin, T. Sun, J. Wang, And S. Zhang, Sharp L2 error estimates and superconvergence of mixed finite element methods for non-Fickian flows in porous media, SIAM J. Numer. Anal., 40 (2002), 1538-1560.

[20] M. Gurtin And A. Pipkin, A general theory of heat conduction with finite wave speeds, Arch. Rational Mech. Anal., 31 (1968), 113-126.

[21] V. Girault And J. L. Lions, Two-grid finite-element schemes for the steady Navier-Stokes problem in polyhedra. Portugal Math., 58 (2001), 25-57.

[22] V. Girault and J. L. Lions, Two-grid finite-element schemes for the transient Navier-Stokes equations, M2AN 35 (2001), 945-980.

[23] Y. HE AND K. LI, Two-level stabilized finite element methods for the Steady Navier-Stokes problem, Computing, 74 (2005), 337-351.

[24] X. Hu And X. Cheng, Accleration of a two-grid method for eigenvalue problems, Math. Comput. 80 (2011), 1287-1301.

[25] Y. Q. HuAng, Time discretization scheme for an integro-differential equation of parabolic type, J. Comput. Math., 3 (1994), 259-264.

[26] J. Jin, S. Shu And J. Xu, A two-grid discretization method for decoupling systems of partial differential equations, Math. Comp. 75 (2006), 1617-1626. 
[27] W. LAYTON And L. TOBISKA, A two-level method with backtracking for the Navier-Stokes equations, SIAM J. Numer. Anal., 35 (1998), 20352054.

[28] S. Li AND Z. HuAng, Two-grid algorithms for some linear and nonlinear elliptic systems, Computing, 89 (2010), 69-86.

[29] Y. LIN, Galerkin methods for nonlinear parabolic integrodifferential equations with nonlinear boundary conditions, SIAM J. Numer. Anal., 27 (1990), 608-621.

[30] Y. Lin, V. ThoméE And L. Wahlbin, Ritz-Volterra projections onto finite element spaces and applications to integro-differential and related equations, SIAM J. Numer. Anal. 28 (1991), 1047-1070.

[31] J. C. Lopez-MArcos, A difference scheme for a nonlinear partial integrodifferential equation, SIAM J. Numer. Anal. 27 (1990), 20-31.

[32] M. MARIOn AND J. Xu, Error estimates on a new nonlinear Galerkin method based on two-grid finite elements, SIAM J. Numer. Anal. 32 (1995), 1170-1184.

[33] R. K. Miller, An integro-differential equation for rigid heat conductions with memory, J. Math. Anal. Appl., 66 (1978), 313-332.

[34] M. Mu AND J. XU, A two-grid method of a mixed Stokes-Darcy model for coupling fluid flow with porous media flow, SIAM J. Numer. Anal., 45 (2007), 1801-1813.

[35] K. Mustapha, H. Brunner, H. Mustapha, and D. Schotzau, An hp-version discontinuous Galerkin method for integro-differential equations of parabolic type, SIAM J. Numer. Anal., 49 (2011), 1369-1396.

[36] A. K. Pani, G. Fairweather, And R. I. Fernandes, Alternating direction implicit orthogonal spline collocation methods for an evolution equation with a positive-type memory term, SIAM J. Numer. Anal., 46 (2008), 344-364.

[37] A. K. Pani And T. E. Peterson, Finite element methods with numerical quadrature for parabolic integrodifferential equations, SIAM J. Numer. Anal., 33 (1996), 1084-1105. 
[38] X. Qin AND Y. MA, Two-grid scheme for characteristics finite-element solution of nonlinear convection diffusion problems, Appl. Math. Comput., 165 (2005) 419-431.

[39] M. RaYnaL, On some nonlinear problems of diffusion, in Volterra Equations, S. London and 0. Staffans, eds., Lecture Notes in Math., 737, Springer-Verlag, Berlin, New York, 1979, pp. 251-266.

[40] Y. SHANG AND K. WANG, Local and parallel finite element algorithms based on two-grid discretization for the transient Stokes equations, Numer. Algor. 54 (2010), 195-218.

[41] R. K. Sinha, R. E. Ewing, And R. D. Lazarov, Mixed finite element approximations of parabolic integro-differential equations with nonsmooth initial data, SIAM J. Numer. Anal., 47 (2009), 3269-3292.

[42] R. K. Sinha, R. E. Ewing, and R. D. Lazarov, Some new error estimates of a semidiscrete finite volume element method for a parabolic integro-differential equation with nonsmooth initial data, SIAM J. Numer. Anal., 43 (2006), 2320-2343.

[43] I. H. Sloan and V. Thomee, Time discretization of an integrodifferential equation of parabolic type, SIAM J. Numer. Math., 23 (1986), 1052-1061.

[44] T. Tachim Medjo and R. Temam, A two-grid finite difference method for the primitive equations of the ocean, Nonlinear Anal., 69 (2008), 1034-1056.

[45] W. S. Wang, Long-time behaviour of two-grid finite element method for the fully discrete semilinear evolution equations with positive memory, J. Comput. Appl. Math., 250 (2013), 161-174.

[46] L. Wu And M. B. Allen, A two-grid method for mixed finite element solution of reaction-diffusion equations, Numer. Methods Partial Differ. Equ. 15 (199), 317-332.

[47] J. Xu, A new class of iterative methods for nonselfadjoint or indefinite elliptic problems, SIAM J. Numer. Anal. 29 (1992), 303-319. 
[48] J. XU, Some Two-Grid Finite Element Methods, Tech. Report, P.S.U, 1992.

[49] J. XU, A novel two-grid method for semi-linear equations, SIAM J. Sci. Comput. 15 (1994), 231-237.

[50] J. XU, Two-grid finite element discretization techniques for linear and nonlinear PDE, SIAM J. Numer. Anal. 33 (1996), 1759-1777.

[51] J. Xu AND A. Zhou, Local and parallel finite element algorithms based on two-grid discretization for nonlinear problems, Adv. Comput. Math., 14 (2001), 293-327.

[52] J. Xu And A. Zhou, A two-grid discretization scheme for eigenvalue problems, Math. Comput., 70 (2001), 17-25.

[53] J. Xu AND A. Zhou, Local and parallel finite element algorithms for eigenvalue problems, Acta Math. Appl. Sin. Engl. Ser. 18 (2002), 185200.

[54] T. Zhang, Finite element methods for partial differenio-integral equations, Beijing: Science Press, 2009 (in Chinese). 